\title{
Characterization of optimal shapes and masses through Monge-Kantorovich equation
}

Received February 10, 2000 / final version received July 21, 2000

Published online November 8, 2000 - (C) Springer-Verlag \& EMS 2000

\begin{abstract}
We study some problems of optimal distribution of masses, and we show that they can be characterized by a suitable Monge-Kantorovich equation. In the case of scalar state functions, we show the equivalence with a mass transport problem, emphasizing its geometrical approach through geodesics. The case of elasticity, where the state function is vector valued, is also considered. In both cases some examples are presented.
\end{abstract}

\section{Introduction}

The analysis of the behaviour of elastic structures has always been a central problem in Mathematics and in Engineering. Since the beginning of the mathematical theory of elasticity it was possible to consider from a rigorous point of view the problem of finding the structure that, for a given system $f$ of loads, gives the best resistance in terms of minimal compliance. In other words, an elastic structure is optimal if the corresponding displacement $u$ is such that the total work $\int f \cdot u d x$ is minimal. However, even if the setting of the problem does not require particular mathematical tools, only in the last two decades there has been a deep understanding of shape optimization problems from a mathematical point of view. This was mainly due to the dramatic improvement in the field impressed by the powerful theories of homogenization and $\Gamma$-convergence which have been developed meanwhile.

What became clear soon was that in a large number of situations the optimal shape does not exists, and the existence of an optimal solution must be intended only in a relaxed sense. The form of the relaxed optimization problem was first studied (see [21,22]) in the so called scalar case where the physical problem only involves state variables with value in $\mathbf{R}$, like the problem of optimal mixtures of two given conductors. In this case the relaxed solutions have been completely studied, and identified as symmetric matrices with bounded and measurable coefficients, whose eigenvalues satisfy some suitable bounds. A similar result was also obtained in the

G. Bouchitté: Département de Mathématiques, Université de Toulon et du Var, BP 132, 83957 La Garde Cedex, France, e-mail: bouchitte@ univ-tln.fr

G. Buttazzo: Dipartimento di Matematica, Università di Pisa, Via Buonarroti, 2, 56127 Pisa, Italy, e-mail: buttazzo@dm.unipi.it

Mathematics Subject Classification (1991): 49J45, 49K20, 49J20, 90B06, 28A50 
elasticity problem (see for instance [15]) for optimal mixtures of two homogeneous and isotropic materials.

Moreover, in almost all cases which have been considered, the optimal relaxed solution is not isotropic (i.e. the optimal matrix is not of the form $a(x) I$, being $I$ the identity matrix), and this was interpreted by saying that an optimal shape does not exist and minimizing sequences are composed by laminates.

We want to emphasize that the case of optimal elastic structures, or also simply the study of optimal shapes of a given conductor, seem to have an additional difficulty with respect to the problem of optimal mixtures. Indeed, the first correspond to the case of optimal mixtures when one of the two materials (or conductors) has the elasticity constants (or the conductivity coefficient) equal to zero. In this case, due to a lack of uniform ellipticity, it is known that among all possible relaxed problems, obtained as limits of sequences of elliptic problems on classical domains, there are some that are not of local type, and it is not clear if these nonlocal relaxed solutions could be optimal. This interesting direction of research has been developed recently in [3] and [20], and has deep connections with the theory of Dirichlet forms.

Here we adopt a different point of view and we consider, instead of the shape optimization problem, the mass optimization problem which consists in finding the best distribution of a given amount of elastic material, in order to achieve the minimal compliance. The unknown mass distribution is then a nonnegative measure which may vary in the class of admissible choices, with total mass prescribed, and support possibly constrained in a given design region. Dealing with general measures pushed us to develop in [5] a general framework of variational calculus on measures, bases on a new notion of tangent bundle for a measure, which includes and unifies the classical cases of low dimensional manifolds (membranes, string, junctions, .... ).

The phenomenon of appearance of low dimensional network structures was already remarked (see [1]) in the cases of optimal mixtures of two materials, when the percentage of the strong one tends to zero. Moreover, because of capacitary arguments, concentrated loads are forbidden in the classical framework, but they become admissible as soon as we allow the conductivity coefficient to be singular, or more generally a measure. Then in the framework of our mass optimization problems, we are allowed to consider the general case when for a load we take a given measure.

A first result is that we obtain the existence of an optimal mass distribution for which the elastic compliance is minimal. This optimal measure may present the interesting feature to be composed by terms of different dimensions. Moreover, we characterize these optimal solutions by means of a generalized version of the Monge-Kantorovich partial differential equation which describes the mass transfer problem. A first announcement of the results we obtained appeared in a short note [6].

The plan of the paper is the following. In Sect. 2 we present the mass optimization problem in a quite general framework and we show the existence of an optimal solution in the class of measures. In Sect. 3 we deduce a necessary and sufficient condition for the optimality that we call Monge-Kantorovich equation 
by analogy with the PDE which occurs in mass transportation problems. Section 4 is fully devoted to the scalar case where an optimal measure can be constructed by means of geodesic transport rays. Finally, in Sect. 5 we treat some examples from elasticity as well as some scalar cases; in this last situation we show how the equivalence with mass transportation problems allows us to obtain the explicit construction of optimal measures also in cases when Dirichlet regions or obstacles are present.

\section{The mass optimization problem}

The optimization problem we are going to describe consists in finding the best distribution of a given total mass in order to minimize the elastic compliance under the action of a given force field. In order to take into account also forces which may concentrate on lower dimensional sets we consider a force field $f \in \mathcal{M}\left(\mathbf{R}^{n} ; \mathbf{R}^{n}\right)$, the class of all $\mathbf{R}^{n}$-valued measures on $\mathbf{R}^{n}$ with finite total variation and with compact support. The class of smooth displacements we consider is the Schwartz space $\mathcal{D}\left(\mathbf{R}^{n} ; \mathbf{R}^{n}\right)$ of $C^{\infty}$ functions with compact support; similarly, the notation $\mathcal{D}^{\prime}\left(\mathbf{R}^{n} ; \mathbf{R}^{n}\right)$ stands for the space of vector valued distributions and, for a given nonnegative measure $\mu, L_{\mu}^{p}\left(\mathbf{R}^{n} ; \mathbf{R}^{d}\right)$ denotes the space of $p$-integrable functions with respect to $\mu$ with values in $\mathbf{R}^{d}$.

For a given displacement $u: \mathbf{R}^{n} \rightarrow \mathbf{R}^{n}$ we denote by $j(D u)$ the stored elastic energy density associated to $u$ and we assume:

(2.1) $j$ is convex;

(2.2) $j$ is positively $p$-homogeneous, with $p>1$;

(2.3) $j(z)=j\left(z^{s y m}\right)$ where $z^{\text {sym }}$ is the symmetric part of $z$;

(2.4) there exist two positive constants $\alpha_{1}$ and $\alpha_{2}$ such that

$$
\alpha_{1}\left|z^{s y m}\right|^{p} \leq j(z) \leq \alpha_{2}\left|z^{s y m}\right|^{p} \quad \forall z \in \mathbf{R}^{n \times n} .
$$

For instance, in the case of a homogeneous isotropic linearly elastic material, the function $j$ is given by

$$
j(z)=\beta\left|z^{s y m}\right|^{2}+\frac{\alpha}{2}\left|\operatorname{tr}\left(z^{s y m}\right)\right|^{2}
$$

where $\alpha$ and $\beta$ are the so called Lamé constants.

It is convenient to introduce the convex 1-homogeneous function

$$
\rho(z)=\inf \{t>0: j(z / t) \leq 1 / p\}
$$

by the homogeneity of $j$ we have

$$
j(z)=\frac{1}{p}(\rho(z))^{p} .
$$

Thus, for a given mass distribution $\mu$ the stored elastic energy of a smooth displacement $u \in \mathcal{D}\left(\mathbf{R}^{n} ; \mathbf{R}^{n}\right)$ is given by

$$
J(\mu, u)=\int j(D u) d \mu=\frac{1}{p} \int(\rho(D u))^{p} d \mu
$$


so that the total energy is

$$
J(\mu, u)-\langle f, u\rangle .
$$

Sometimes we write $e(u)$ instead of $(D u)^{s y m}$, so that the stored energy functional can be also written as

$$
J(\mu, u)=\int j(e(u)) d \mu=\frac{1}{p} \int(\rho(e(u)))^{p} d \mu .
$$

The scalar product between $n \times n$ matrices is defined by

$$
z: \xi=\sum_{i, j=1}^{n} z_{i j} \xi_{i j}
$$

in this way we can also introduce the Fenchel conjugate of $j$

$$
j^{*}(z)=\sup \left\{z: \xi-j(\xi): \xi \in \mathbf{R}^{n \times n}\right\}
$$

and the polar function associated to $\rho$ :

$$
\rho^{0}(z)=\sup \{z: \xi: \rho(\xi) \leq 1\} .
$$

It is then easy to obtain the equality

$$
j^{*}(z)=\frac{1}{p^{\prime}}\left(\rho^{0}(z)\right)^{p^{\prime}} \quad \forall z \in \mathbf{R}^{n \times n} .
$$

We consider mass distributions $\mu$ which are nonnegative measures on $\mathbf{R}^{n}$ whose support is contained in the so called design region which is a given closed subset $K$ of $\mathbf{R}^{n}$. It should also be noticed that the problem above is a variational model which describes the behaviour of light structures, where the force due to their own weight can be neglected. Finally, in order to take into account possibly prescribed Dirichlet boundary conditions, we denote by $\mathcal{U}$ the set of smooth admissible displacements, which we assume to be a given convex cone of $\mathcal{D}\left(\mathbf{R}^{n} ; \mathbf{R}^{n}\right)$. Therefore, the infimum

$$
\mathcal{E}(\mu)=\inf \left\{\int j(D u) d \mu-\langle f, u\rangle: u \in \mathcal{U}\right\}
$$

can be considered as the energy associated to the mass distribution $\mu$. The compliance $\mathcal{C}(\mu)$ is then defined as

$$
\mathcal{C}(\mu)=-\mathcal{E}(\mu)
$$

It must be noticed that we may have $\mathcal{C}(\mu)=+\infty$ for some measures $\mu$; this happens for instance in the case $\mathcal{U}=\mathcal{D}\left(\mathbf{R}^{n} ; \mathbf{R}^{n}\right)$ when the force field $f$ concentrates on sets of dimension smaller than $n-1$ and the mass distribution $\mu$ is the Lebesgue measure. However, these "singular" measures $\mu$ are ruled out from our discussion because we look for the minimization of the compliance $\mathcal{C}(\mu)$. Indeed, we consider the optimization problem

$$
\min \left\{\mathcal{C}(\mu): \mu \in \mathcal{M}^{+}\left(\mathbf{R}^{n}\right), \int d \mu=m, \operatorname{spt} \mu \subset K\right\}
$$


where the total amount of mass $m$ is prescribed, as well as the design region $K$. Our goal is to obtain an existence result for problem (2.7) and to characterize its solutions by means of necessary and sufficient conditions of optimality (that we shall call in next section Monge-Kantorovich equation).

It is convenient to introduce the polar cone of distributions

$$
\mathcal{U}^{0}=\left\{T \in \mathcal{D}^{\prime}\left(\mathbf{R}^{n} ; \mathbf{R}^{n}\right):\langle T, u\rangle \leq 0 \quad \forall u \in \mathcal{U}\right\}
$$

then by standard duality arguments (see for instance [12]) the compliance $\mathcal{C}(\mu)$ can be written in the form

$$
\mathcal{C}(\mu)=\inf \left\{\int j^{*}(\sigma) d \mu: \sigma \in L_{\mu}^{p^{\prime}}\left(\mathbf{R}^{n} ; \mathbf{R}^{n \times n}\right), f+\operatorname{div}(\sigma \mu) \in \mathcal{U}^{0}\right\} .
$$

It is straightforward that the infimum in (2.8) is actually a minimum as soon as $\mathcal{C}(\mu)$ is finite.

Let us introduce now the quantity

$$
I(f, \mathcal{U}, K)=\sup \{\langle f, u\rangle: u \in \mathcal{U}, j(D u) \leq 1 / p \text { on } K\}
$$

which can be related to a locking material approach (see [11]).

Proposition 2.1. For every nonnegative measure $\mu$ with $\int d \mu=m$ and spt $\mu \subset K$ we have

$$
\mathcal{C}(\mu) \geq \frac{(I(f, \mathcal{U}, K))^{p^{\prime}}}{p^{\prime} m^{1 /(p-1)}} .
$$

Proof. If $\mathcal{C}(\mu)=+\infty$ the inequality is trivial. If $\mathcal{C}(\mu)$ is finite the infimum in (2.8) is a minimum; let us denote by $\sigma$ a solution: then by Fenchel inequality we have for every $u \in \mathcal{U}$

$$
\mathcal{C}(\mu)=\int j^{*}(\sigma) d \mu \geq \int \sigma: D u d \mu-\int j(D u) d \mu .
$$

Therefore, since $f+\operatorname{div}(\sigma \mu) \in \mathcal{U}^{0}$, we obtain

$$
\int \sigma: D u d \mu=-\langle\operatorname{div}(\sigma \mu), u\rangle \geq\langle f, u\rangle
$$

so that

$$
\mathcal{C}(\mu) \geq\langle f, u\rangle-\int j(D u) d \mu \quad \forall u \in \mathcal{U}
$$

Since $\mathcal{U}$ is a cone and $j$ is positively $p$-homogeneous, we also have

$$
\mathcal{C}(\mu) \geq t\langle f, u\rangle-t^{p} \int j(D u) d \mu \quad \forall u \in \mathcal{U}, \forall t \geq 0 .
$$


Hence, taking the supremum over all $u \in \mathcal{U}$ with $j(D u) \leq 1 / p$ on $K$ we deduce

$$
\mathcal{C}(\mu) \geq t I(f, \mathcal{U}, K)-t^{p} \frac{m}{p}
$$

A further supremum over all $t \geq 0$ finally gives the desired inequality (2.10).

We want to show now that in (2.10) the equality is actually attained for some measure $\mu$; this will provide, as a consequence, an existence result for the mass optimization problem (2.7).

Proposition 2.2. There exists a nonnegative measure $\mu$ with $\int d \mu=m$ and spt $\mu \subset K$ such that

$$
\mathcal{C}(\mu) \leq \frac{(I(f, \mathcal{U}, K))^{p^{\prime}}}{p^{\prime} m^{1 /(p-1)}}
$$

Proof. We have

$$
I(f, \mathcal{U}, K)=\sup \{\langle f, u\rangle: u \in \mathcal{U}, \rho(D u) \leq 1 \text { on } K\}
$$

and, again by duality arguments, we can write

$$
I(f, \mathcal{U}, K)=\inf \left\{\int \rho^{0}(\lambda): \lambda \in \mathcal{M}\left(\mathbf{R}^{n} ; \mathbf{R}^{n \times n}\right), \operatorname{spt} \lambda \subset K, f+\operatorname{div} \lambda \in \mathcal{U}^{0}\right\}
$$

where the integral is intended in the sense of convex functionals on the space of measures (see for instance [17,7]). As before, as soon as $I(f, \mathcal{U}, K)$ is finite, the infimum in (2.12) is a minimum. If we denote by $\lambda$ a solution and set

$$
\mu=\frac{m}{I(f, \mathcal{U}, K)} \rho^{0}(\lambda)
$$

by Radon-Nikodym theorem we obtain that $\lambda=\sigma \mu$ for a suitable $\sigma \in L_{\mu}^{p^{\prime}}\left(\mathbf{R}^{n} ; \mathbf{R}^{n \times n}\right)$. We have spt $\mu \subset K, \int d \mu=m, f+\operatorname{div}(\sigma \mu) \in \mathcal{U}^{0}$, and $\rho^{0}(\sigma)=I(f, \mathcal{U}, K) / m$ $\mu$-a.e., so that by (2.8)

$$
\mathcal{C}(\mu) \leq \int j^{*}(\sigma) d \mu=\frac{1}{p^{\prime}} \int\left(\rho^{0}(\sigma)\right)^{p^{\prime}} d \mu=\frac{(I(f, \mathcal{U}, K))^{p^{\prime}}}{p^{\prime} m^{1 /(p-1)}} .
$$

Summarizing, we have proved the following result.

Theorem 2.3. Assume that $I(f, \mathcal{U}, K)$ is finite. Then the following facts hold:

i) The mass optimization problem (2.7) admits a solution $\mu$ and we have

$$
\mathcal{C}(\mu)=\frac{(I(f, \mathcal{U}, K))^{p^{\prime}}}{p^{\prime} m^{1 /(p-1)}}
$$


ii) If $\mu$ is a solution of the mass optimization problem (2.7) then one has

$$
I(f, \mathcal{U}, K)=\min \left\{\int \rho^{0}(\sigma) d \mu: \sigma \in L_{\mu}^{1}\left(\mathbf{R}^{n} ; \mathbf{R}^{n \times n}\right), f+\operatorname{div}(\sigma \mu) \in \mathcal{U}^{0}\right\}
$$

and every optimal $\sigma$ in (2.13) verifies

$$
\rho^{0}(\sigma)=\frac{I(f, \mathcal{U}, K)}{m} \quad \mu \text {-almost everywhere. }
$$

iii) Conversely, if $\lambda$ is a solution for (2.12), then the nonnegative measure $\mu:=$ $\frac{m}{I(f, \mathcal{U}, K)} \rho^{0}(\lambda)$ is optimal for (2.7).

We will discover in the next section that the field $\sigma$ solution of (2.13) is in fact related to the gradient of the optimal displacement $u$ (for (2.6)) through a constitutive equation involving some notion of tangent space to $\mu$.

Let us emphasize some few facts about what we obtained in this section. A remarkable issue is that by Theorem 2.3 ii) and iii) the optimal measures $\mu$ can be deduced from solutions of problem (2.12), hence they do not depend on the growth exponent $p$ of the energy density $j$ but only on the convex level set $\left\{\xi \in \mathbf{R}^{n \times n}: \rho(\xi) \leq 1\right\}$. Moreover, when $\mu$ is an optimal mass distribution, by (2.14) the associated stress density $j^{*}(\sigma)=\frac{1}{p^{\prime}}\left(\rho^{0}(\sigma)\right)^{p^{\prime}}$ is constant (the same will be true for the $\mu$-strain energy density, as defined in Sect. 3).

According to the independence seen above of optimal measures $\mu$ of the growth exponent $p$, it seems natural to consider problems (2.9) and (2.12) as the candidates for being the limit as $p \rightarrow+\infty$ of the primal strain problem (2.6) and the dual stress problem (2.8) respectively. This idea, heuristically stated above, has been used in [18] for obtaining numerical approximations of solutions to several shape optimization problems.

\section{The Monge-Kantorovich equation}

In this section we discuss the existence of a relaxed solution for problem (2.9) and the related necessary and sufficient conditions of optimality. This will produce what we call Monge-Kantorovich equation. From now on, for simplicity, we assume that:

- $K$ is a closure of a smooth connected bounded open subset $\Omega$ of $\mathbf{R}^{n}$;

- $\mathcal{U}=\left\{u \in \mathcal{D}\left(\mathbf{R}^{n} ; \mathbf{R}^{n}\right): u=0\right.$ on $\left.\Sigma\right\}$ where $\Sigma$ is a closed subset of $\bar{\Omega}$.

The quantity $I(f, \mathcal{U}, K)$ will be then denoted by $I(f, \Sigma, \Omega)$. We remark that under the assumptions above the class $\mathcal{U}^{0}$ turns out to be

$$
\mathcal{U}^{0}=\left\{T \in \mathcal{D}^{\prime}\left(\mathbf{R}^{n} ; \mathbf{R}^{n}\right): \operatorname{spt} T \subset \Sigma\right\} .
$$

It is convenient to introduce now the class $\operatorname{Lip}_{1, \rho}(\Omega, \Sigma)$ as the closure, in $C\left(\bar{\Omega} ; \mathbf{R}^{n}\right)$, of the set $\left\{u \in \mathcal{D}\left(\mathbf{R}^{n} ; \mathbf{R}^{n}\right): \rho(D u) \leq 1\right.$ on $\Omega, u=0$ on $\left.\Sigma\right\}$. It has to be noticed that 
when $\rho(z) \geq|z|$ then every function in $\operatorname{Lip}_{1, \rho}(\Omega)$ is locally Lipschitz continuous on $\Omega$; on the other hand, if $\rho(z)=\left|z^{\text {sym }}\right|$ this is no more true, due to the lack of Korn inequality for $p=+\infty$ (see for instance [10]).

We define the relaxed formulation of problem (2.9) as

$$
\sup \left\{\langle f, u\rangle: u \in \operatorname{Lip}_{1, \rho}(\Omega, \Sigma)\right\}
$$

and the finite dimensional linear space of all rigid displacements vanishing on $\Sigma$

$$
\mathcal{R}_{\Sigma}=\left\{u(x)=A x+b: b \in \mathbf{R}^{n}, A \in \mathbf{R}_{\text {skew }}^{n \times n}, u=0 \text { on } \Sigma\right\} .
$$

Proposition 3.1. Let $\Omega$ and $\Sigma$ be defined as above. Then the supremum in problem (2.9) is finite if and only if

$$
\langle f, u\rangle=0 \quad \forall u \in \mathcal{R}_{\Sigma} .
$$

In this case, problem (3.1) admits a solution and

$$
\sup (2.9)=\max (3.1)
$$

Proof. The first assertion is a well known fact (see for instance [2]). To conclude the proof we just need to prove the existence of a solution of problem (3.1) under condition (3.2). Let $q>n$ and let $P_{\Sigma}$ be any linear continuous projector from $L^{q}\left(\Omega ; \mathbf{R}^{n}\right)$ into the closed finite dimensional subspace $\mathcal{R}_{\Sigma}$; arguing by contradiction and using the Korn inequality and the Rellich compactness theorem it is easy to obtain the inequality

$$
\left\|u-P_{\Sigma} u\right\|_{L^{q}(\Omega)} \leq C\left[\|e(u)\|_{L^{q}(\Omega)}+\int_{\Sigma}|u| d x\right] .
$$

Let now $\left(u_{h}\right)$ be a maximizing sequence for problem (2.9); thanks to assumption (3.2) we may assume that $P_{\Sigma}\left(u_{h}\right)=0$ for every $h$. By using Korn's inequality again and (3.3) we obtain that $\left(u_{h}\right)$ is relatively compact in $C(\bar{\Omega})$ and so all its cluster points belong to $\operatorname{Lip}_{1, \rho}(\Omega, \Sigma)$, vanish on $\Sigma$, and solve (3.1).

In order to well define the optimality conditions for problem (2.7), we need to introduce the function space of displacements of finite energy related to a general measure $\mu$. Since the dimension of the measure $\mu$ is not a priori fixed, we have to develop a general scheme which encompasses the theories of elastic membranes, elastic strings, junctions of multidimensional structures, etc. In particular, we have to generalize the usual notions of tangential gradient and tangential strain which naturally occur when one deals with variational problems on smooth surfaces. Following a scheme similar to the one already used in [5] and in [6], given a measure $\mu$ and an open subset $U$ of $\mathbf{R}^{n}$ we define the space of admissible stresses

$$
X_{\mu}^{p^{\prime}}\left(U ; \mathbf{R}_{s y m}^{n \times n}\right)=\left\{\sigma \in L_{\mu}^{p^{\prime}}\left(U ; \mathbf{R}_{s y m}^{n \times n}\right): \operatorname{div}(\sigma \mu) \in \mathcal{M}\left(\mathbf{R}^{n} ; \mathbf{R}^{n}\right)\right\} .
$$


It turns out that this space is local in the sense that

$$
\phi \sigma \in X_{\mu}^{p^{\prime}}\left(U ; \mathbf{R}_{s y m}^{n \times n}\right) \quad \forall \phi \in \mathcal{D}\left(\mathbf{R}^{n}\right), \forall \sigma \in X_{\mu}^{p^{\prime}}\left(U ; \mathbf{R}_{s y m}^{n \times n}\right),
$$

so that its closure in $L_{\mu}^{p^{\prime}}$ can be written as

$$
\left\{\sigma \in L_{\mu}^{p^{\prime}}\left(U ; \mathbf{R}_{s y m}^{n \times n}\right): \sigma(x) \in \mathcal{M}_{\mu}(x)\right\}
$$

where

$$
M_{\mu}(x)=\mu-\operatorname{ess} \bigcup\left\{\sigma(x): \sigma \in X_{\mu}^{p^{\prime}}\left(U ; \mathbf{R}_{s y m}^{n \times n}\right)\right\} .
$$

Here $\mu$ - ess stands for the $\mu$ essential union, according to Bouchitté and Valadier [7], see also [5]. If we denote by $P_{\mu}(x)$ the orthogonal projector on $M_{\mu}(x)$ with respect to the usual scalar product on matrices, for every function $u \in \mathcal{D}\left(U ; \mathbf{R}^{n}\right)$ we may then define the tangential strain $e_{\mu}(u)$ as

$$
e_{\mu}(u)(x)=P_{\mu}(x) D u(x)
$$

We notice that for $\mu$-a.e. $x \in U$ we have $e_{\mu}(u)(x) \in M_{\mu}(x)$. In the case of a smooth manifold $S$ in $\mathbf{R}^{n}$ of dimension $k \leq n$, which corresponds to the measure $\mu=\mathcal{H}^{k}\left\llcorner S\right.$, it is easy to obtain that the tangential strain $e_{\mu}$ defined above is given by

$$
e_{\mu}(u)(x)=\mathcal{P}_{S}(x)(D u)^{s y m} \mathcal{P}_{S}(x)
$$

where $\mathcal{P}_{S}(x)$ is the orthogonal projection on the tangent space to $S$ at $x$, and $M_{\mu}(x)$ is the set

$$
M_{\mu}(x)=\left\{\mathcal{P}_{S}(x) \xi \mathcal{P}_{S}(x): \xi \in \mathbf{R}_{s y m}^{n \times n}\right\} .
$$

It turns out that the definition above of $e_{\mu}$ only depends on the equivalence class $\mu$-a.e. of $u$, as the following lemma shows.

Lemma 3.2. Let $u_{h} \in \mathcal{D}\left(U ; \mathbf{R}^{n}\right)$ be a sequence such that $u_{h} \rightarrow 0$ uniformly on $U$ and $e_{\mu}\left(u_{h}\right) \rightarrow \xi$ in $L_{\mu}^{p}\left(U ; \mathbf{R}_{s y m}^{n \times n}\right)$. Then $\xi=0$.

Proof. As $e_{\mu}\left(u_{h}\right)(x)$ belongs to the closed subspace $M_{\mu}(x)$, we also have $\xi(x) \in$ $M_{\mu}(x)$ for $\mu$-a.e. $x \in U$. Let now $\sigma \in X_{\mu}^{p^{\prime}}\left(U ; \mathbf{R}_{s y m}^{n \times n}\right)$; then

$$
\int \xi: \sigma d \mu=\lim _{h \rightarrow+\infty} \int e_{\mu}\left(u_{h}\right): \sigma d \mu=-\lim _{h \rightarrow+\infty}\left\langle u_{h}, \operatorname{div}(\sigma \mu)\right\rangle=0
$$

since $\operatorname{div}(\sigma \mu)$ is a bounded measure and $u_{h} \rightarrow 0$ uniformly. The equality above can be extended to all $\sigma$ in the closure of $X_{\mu}^{p^{\prime}}\left(U ; \mathbf{R}_{s y m}^{n \times n}\right)$, that is to all $\sigma \in L_{\mu}^{p^{\prime}}\left(U ; \mathbf{R}_{s y m}^{n \times n}\right)$ with $\sigma(x) \in M_{\mu}(x)$, and this clearly concludes the proof.

Thanks to the lemma above the linear operator

$$
u \in \mathcal{D}\left(U ; \mathbf{R}^{n}\right) \mapsto e_{\mu}(u) \in L_{\mu}^{p}\left(U ; \mathbf{R}_{s y m}^{n \times n}\right)
$$


is closable as an operator from $C\left(\bar{U} ; \mathbf{R}^{n}\right)$ into $L_{\mu}^{p}\left(U ; \mathbf{R}_{s y m}^{n \times n}\right)$. In the following we still denote by $e_{\mu}$ the closed operator from $C\left(\bar{U} ; \mathbf{R}^{n}\right)$ into $L_{\mu}^{p}\left(U ; \mathbf{R}_{s y m}^{n \times n}\right)$ which extends the tangential strain, in the sense of proposition above.

Now we can define the Banach space of all finite energy displacements $\mathcal{D}_{0, \mu}^{1, p}(U)$ as the domain of the operator $e_{\mu}$ endowed with the norm

$$
\|u\|_{\mathcal{D}_{0, \mu}^{1, p}(U)}=\|u\|_{C(\bar{U})}+\left\|e_{\mu}(u)\right\|_{L_{\mu}^{p}(U)} .
$$

Remark 3.3. We notice that if $\left(u_{h}\right)$ is a sequence which converges to $u$ uniformly on $U$ and such that $e_{\mu}\left(u_{h}\right)$ is bounded in $L_{\mu}^{p}$, then by the closedness of the operator $e_{\mu}$ and the reflexivity of $L_{\mu}^{p}\left(U ; \mathbf{R}^{n \times n}\right)$ we have that $u \in \mathcal{D}_{0, \mu}^{1, p}(U)$ and $e_{\mu}\left(u_{h}\right) \rightarrow e_{\mu}(u)$ weakly in $L_{\mu}^{p}\left(U ; \mathbf{R}^{n \times n}\right)$.

Since $\mathcal{D}_{0, \mu}^{1, p}(U)$ is also the completion of $\mathcal{D}\left(U ; \mathbf{R}^{n}\right)$ with respect to the norm above, the integration by parts formula

$$
\int e_{\mu}(u): \sigma d \mu=-\langle u, \operatorname{div}(\sigma \mu)\rangle
$$

holds for every $u \in \mathcal{D}_{0, \mu}^{1, p}(U)$ and every $\sigma \in X_{\mu}^{p^{\prime}}\left(U ; \mathbf{R}_{s y m}^{n \times n}\right)$. Notice that for all such pairs $(u, \sigma)$ we have

$$
e_{\mu}(u)(x) \in M_{\mu}(x), \quad \sigma(x) \in M_{\mu}(x) \quad \text { for } \mu \text {-a.e. } x \in U .
$$

As a variant of the argument used in [5] we obtain the relaxed form of the stored energy functional $J(\mu, u)$

$$
\begin{aligned}
\bar{J}(\mu, u) & =\inf \left\{\liminf _{h \rightarrow+\infty} J\left(\mu, u_{h}\right): u_{h} \rightarrow u \text { uniformly, } u_{h} \in \mathcal{D}\left(U ; \mathbf{R}^{n}\right)\right\} \\
& = \begin{cases}\int_{U} j_{\mu}\left(x, e_{\mu}(u)\right) d \mu & \text { if } u \in \mathcal{D}_{0, \mu}^{1, p}(U) \\
+\infty & \text { otherwise }\end{cases}
\end{aligned}
$$

where

$$
j_{\mu}(x, z)=\inf \left\{j(z+\xi): \xi \in\left(M_{\mu}(x)\right)^{\perp}\right\} .
$$

Remark 3.4. Let us specify the spaces $\mathcal{D}_{0, \mu}^{1, p}(U)$, the operators $e_{\mu}$, and the energy densities $j_{\mu}$ in some particular situations.

a) If $\mu$ is the Lebesgue measure over some regular open subset $U$ of $\mathbf{R}^{n}$, then $e_{\mu}(u)$ coincides with the usual strain tensor $(D u)^{s y m}$ and $j_{\mu}(x, z)=j(z)$ for a.e. $x \in U$. Moreover, thanks to Korn inequality, $\mathcal{D}_{0, \mu}^{1, p}(U)=W_{0}^{1, p}\left(U ; \mathbf{R}^{n}\right)$. 
b) If $\mu$ is the 2-dimensional Hausdorff measure on a smooth surface $S$ of $\mathbf{R}^{3}$ having $v(x)$ as a unit normal vector, then $e_{\mu}(u)$ represents the usual tangential strain

$$
e_{\mu}(u)=(I-v \otimes v) e(u)(I-v \otimes v)
$$

and $j_{\mu}(x, z)$ gives, in the case (2.5), the well known membrane stored energy density (see [5])

$$
j_{\mu}\left(x, e_{\mu}(u)\right)=\frac{\alpha \beta}{\alpha+2 \beta}\left|\operatorname{tr} e_{\mu}(u)\right|^{2}+\beta\left|e_{\mu}(u)\right|^{2} .
$$

c) In the case of a measure $\mu$ given by the 1-dimensional Hausdorff measure on a smooth curve $\Gamma$ with unit tangent vector $\tau$ then $e_{\mu}(u)$ represents the tangential deformation $D u \tau \cdot \tau$ and $j_{\mu}(x, z)$ is, in the case (2.5), simply proportional to $|D u \tau \cdot \tau|^{2}$ (see [5]) and precisely

$$
j_{\mu}\left(x, e_{\mu}(u)\right)=\frac{\beta(3 \alpha+2 \beta)}{2(\alpha+\beta)}|D u \tau \cdot \tau|^{2} .
$$

Remark 3.5. We can actually define the initial energy starting from displacements which belong to $\left\{u \in C^{1}\left(\bar{U} ; \mathbf{R}^{n}\right): u=0\right.$ on $\left.\partial U\right\}$ instead of $\mathcal{D}\left(U ; \mathbf{R}^{n}\right)$. Indeed, by the same approximation procedure of lemma below, we can show that every such function belongs to $\mathcal{D}_{0, \mu}^{1, p}(U)$ and satisfies $\mu$-a.e. the equality $e_{\mu}(u)=P_{\mu}(x) D u$. Therefore we obtain the same expression (3.6) for the relaxed functional

$$
\bar{J}(\mu, u)=\inf \left\{\begin{array}{l}
\liminf _{h \rightarrow+\infty} J\left(\mu, u_{h}\right): u_{h} \rightarrow u \text { uniformly, } \\
u_{h} \in C^{1}\left(\bar{U} ; \mathbf{R}^{n}\right), u_{h}=0 \text { on } \partial U
\end{array}\right\} .
$$

Let us now go back to the optimization problem (3.1).

Lemma 3.6. For every measure $\mu \in \mathcal{M}^{+}(\bar{\Omega})$ we have the inclusion

$$
\operatorname{Lip}_{1, \rho}(\Omega, \Sigma) \subset\left\{u \in \mathcal{D}_{0, \mu}^{1, p}\left(\mathbf{R}^{n} \backslash \Sigma\right): j_{\mu}\left(x, e_{\mu}(u)\right) \leq 1 / p \mu \text {-a.e. on } \mathbf{R}^{n} \backslash \Sigma\right\} \text {. }
$$

Proof. Let $u \in \operatorname{Lip}_{1, \rho}(\Omega, \Sigma)$ and let $\left(u_{h}\right)$ be an approximating sequence in $\mathcal{D}\left(\mathbf{R}^{n} ; \mathbf{R}^{n}\right)$ such that

$$
u_{h} \rightarrow u \text { uniformly on } \Omega, \quad \rho\left(D u_{h}\right) \leq 1 \text { on } \Omega, \quad u_{h}=0 \text { on } \Sigma .
$$

Take $v_{h}=\alpha_{h} u_{h}$ where $\alpha_{h} \in C^{\infty}\left(\mathbf{R}^{n} ;[0,1]\right)$ satisfies:

$$
\alpha_{h}(x)=0 \text { if } \operatorname{dist}(x, \Sigma)<1 / h, \quad \alpha_{h} \uparrow 1 \text { on } \mathbf{R}^{n} \backslash \Sigma, \quad\left|u_{h}\right|\left|D \alpha_{h}\right| \leq C .
$$

This is possible because $\left(u_{h}\right)$ converges uniformly to $u$ which vanishes on $\Sigma$. Then $v_{h} \in \mathcal{D}\left(\mathbf{R}^{n} \backslash \Sigma ; \mathbf{R}^{n}\right)$ and, since $\alpha_{h}$ converges uniformly to 1 on every compact subset of $\mathbf{R}^{n} \backslash \Sigma$, we have $v_{h} \rightarrow u$ in $C\left(\bar{\Omega} ; \mathbf{R}^{n}\right)$. In addition, from the equality

$$
e\left(v_{h}\right)=\alpha_{h} e\left(u_{h}\right)+\left(u_{h} \otimes D \alpha_{h}\right)^{s y m}
$$


we deduce that $\left(e_{\mu}\left(v_{h}\right)\right)$ is uniformly bounded in $L_{\mu}^{p}\left(\mathbf{R}^{n} \backslash \Sigma ; \mathbf{R}^{n \times n}\right)$. The closedness of the operator $e_{\mu}$ implies (see Remark 3.3) that $u \in \mathcal{D}_{0, \mu}^{1, p}\left(\mathbf{R}^{n} \backslash \Sigma\right.$ ) and that the weak limit of $\left(e_{\mu}\left(v_{h}\right)\right)$ coincides with $e_{\mu}(u)$.

To prove that $j_{\mu}\left(x, e_{\mu}(u) \leq 1 / p \mu\right.$-a.e. on $\mathbf{R}^{n} \backslash \Sigma$ we apply the relaxation formula (3.6) to the sequence $\left(u_{h}\right)$ and we use a localization argument to obtain that

$$
\int_{U} j_{\mu}\left(x, e_{\mu}(u)\right) d \mu \leq \liminf _{h \rightarrow+\infty} \int_{U} j_{\mu}\left(x, e_{\mu}\left(u_{h}\right)\right) d \mu \leq \frac{1}{p} \mu(U)
$$

holds actually for every open subset $U$ of $\mathbf{R}^{n} \backslash \Sigma$. This gives the inequality

$$
j_{\mu}\left(x, e_{\mu}(u)\right) \leq 1 / p \mu \text {-a.e. on } \mathbf{R}^{n} \backslash \Sigma \text {. }
$$

Proposition 3.7. Let $\mu$ be a solution of the mass optimization problem (2.7). Then $\mu$ does not charge the Dirichlet region $\Sigma$, i.e. $\mu(\Sigma)=0$. Moreover, if $u$ and $\sigma$ are solutions of problems (3.1) and (2.13) respectively, then

$$
\begin{gathered}
\frac{m}{I} \sigma(x) \in \partial j_{\mu}\left(x, e_{\mu}(u)\right) \quad \mu \text {-a.e. on } \mathbf{R}^{n} \\
j_{\mu}\left(x, e_{\mu}(u)\right)=1 / p \quad \text { and } \quad j_{\mu}^{*}(x, m \sigma / I)=1 / p^{\prime} \quad \text { M-a.e. on } \mathbf{R}^{n}
\end{gathered}
$$

where $I=I(f, \Sigma, \Omega)$ and $\partial j_{\mu}(x, \cdot)$ denotes the subdifferential of the convex function $j_{\mu}(x, \cdot)$. Consequently, $\sigma$ is a solution of the dual problem (2.8) and the rescaled function $(I / m)^{1 /(p-1)} u$ is a solution of the relaxed problem

$$
\min \left\{\bar{J}(\mu, v)-\langle f, v\rangle: v \in \mathcal{D}_{0, \mu}^{1, p}\left(\mathbf{R}^{n} \backslash \Sigma\right)\right\}
$$

where the functional $\bar{J}$ is defined in (3.6).

Proof. By Lemma 3.6 the function $u$ belongs to $\mathcal{D}_{0, \mu}^{1, p}\left(\mathbf{R}^{n} \backslash \Sigma\right)$. On the other hand, $f+\operatorname{div}(\sigma \mu)=0$ on $\mathbf{R}^{n} \backslash \Sigma$ and therefore $\sigma \in X_{\mu}^{p^{\prime}}\left(\mathbf{R}^{n} \backslash \Sigma ; \mathbf{R}_{\text {sym }}^{n \times n}\right)$. The integration by parts formula (3.4) together with Fenchel inequality then gives

$$
I=\langle f, u\rangle=\int_{\mathbf{R}^{n} \backslash \Sigma} e_{\mu}(u): \sigma d \mu \leq \frac{I}{m} \int_{\mathbf{R}^{n} \backslash \Sigma}\left[j_{\mu}\left(x, e_{\mu}(u)\right)+j_{\mu}^{*}\left(x, \frac{m \sigma}{I}\right)\right] d \mu .
$$

Noticing that $j_{\mu}^{*}(x, \cdot)=j^{*}(\cdot)$ on $M_{\mu}(x)$ (see the definitions of $j_{\mu}$ and $M_{\mu}$ ) and that $\sigma(x) \in M_{\mu}(x)$ (see (3.5)), by Theorem 2.3 we obtain

$$
j_{\mu}^{*}\left(x, \frac{m \sigma}{I}\right)=j^{*}\left(x, \frac{m \sigma}{I}\right)=\frac{1}{p^{\prime}}\left(\frac{m}{I}\right)^{p^{\prime}}\left|\rho^{0}(\sigma)\right|^{p^{\prime}}=\frac{1}{p^{\prime}} .
$$

On the other hand, by Lemma 3.6 we have $j_{\mu}\left(x, e_{\mu}(u)\right) \leq 1 / p$ on $\mathbf{R}^{n} \backslash \Sigma$, so that, dividing by $I$ in (3.11), we have

$$
1 \leq \frac{1}{m} \mu\left(\mathbf{R}^{n} \backslash \Sigma\right)=\frac{1}{m}(m-\mu(\Sigma))
$$


which implies $\mu(\Sigma)=0$. Therefore (3.11) gives

$$
m=\frac{m}{I} \int e_{\mu}(u): \sigma d \mu \leq \int\left[j_{\mu}\left(x, e_{\mu}(u)\right)+j_{\mu}^{*}\left(x, \frac{m \sigma}{I}\right)\right] d \mu \leq m
$$

which implies (3.8) and (3.9).

Summarizing, we have proved that if $\mu$ solves the mass optimization problem (2.7) and $u$ and $\sigma$ are solutions of problems (3.1) and (2.13) respectively, then the triple $(u, m \sigma / I, I \mu / m)$ solves what we call Monge-Kantorovich equation

$$
\left\{\begin{array}{rll}
\text { i) } & f+\operatorname{div}(\sigma \mu)=0 \quad \text { on } \mathbf{R}^{n} \backslash \Sigma \\
\text { ii) } & \sigma \in \partial j_{\mu}\left(x, e_{\mu}(u)\right) \quad \mu \text {-a.e. on } \mathbf{R}^{n} \\
\text { iii }) & u \in \operatorname{Lip}_{1, \rho}(\Omega, \Sigma) \\
\text { iv) } & j_{\mu}\left(x, e_{\mu}(u)\right)=1 / p \quad \mu \text {-a.e. on } \mathbf{R}^{n} \\
\text { v) } & \mu(\Sigma)=0 .
\end{array}\right.
$$

We shall now prove the vice versa. Notice that by conditions i) and iv) the field $\sigma(x)$ (as well as $e_{\mu}(x)$ ) belongs to the subspace $M_{\mu}(x)$ for $\mu$-a.e. $x$. Moreover, as announced at the end of Sect. 2, it turns out that by condition iv) the tangential strain energy $j_{\mu}\left(x, e_{\mu}(u)\right)$ is constant for optimal mass distributions $\mu$.

Lemma 3.8. Let $h: \mathbf{R}^{d} \rightarrow[0,+\infty]$ be a convex positively $p$-homogeneous function and let $v, w \in \mathbf{R}^{d}$ be such that $h(v)=1 / p$ and $w \in \partial h(v)$. Then $h^{*}(w)=1 / p^{\prime}$ and $v \cdot w=1$.

Proof. We have

$$
h^{*}(w)=v \cdot w-h(v)=\sup \left\{t v \cdot w-t^{p} h(v): t>0\right\} .
$$

Hence the supremum above is achieved for $t=1$, which implies that $v \cdot w=$ $p h(v)=1$. Thus

$$
h^{*}(w)=(1-p) h(v)=1 / p^{\prime} .
$$

Theorem 3.9. If the triple $(u, \sigma, \mu)$ solves the Monge-Kantorovich equation (3.12), then $u$ is a solution of problem (3.1) and the measure $m \mu / I$ is a solution of the mass optimization problem (2.7). Moreover, $(I / m)^{1 /(p-1)} u$ is a solution of the relaxed displacement problem (3.10) and $I \sigma / m$ is a solution of the stress problem (2.8), both related to the measure $m \mu / I$.

Proof. We prove first that $u$ is a solution of (3.1) and that $\mu\left(\mathbf{R}^{n}\right)=I$. Let $\left(u_{h}\right)$ be a sequence in $\mathcal{D}\left(\mathbf{R}^{n} ; \mathbf{R}^{n}\right)$ converging to $u$ uniformly on $\Omega$, with $e\left(u_{h}\right)$ uniformly bounded on $\Omega$, and vanishing on $\Sigma$. With an argument similar to the one used in the proof of Lemma 3.6 we may assume that every $u_{h}$ actually vanishes in a neighbourhood of $\Sigma$ (depending on $h$ ). Then we have 


$$
\begin{aligned}
\langle f, u\rangle & =\lim _{h \rightarrow+\infty}\left\langle f, u_{h}\right\rangle=-\lim _{h \rightarrow+\infty}\left\langle\operatorname{div}(\sigma \mu), u_{h}\right\rangle \\
& =\lim _{h \rightarrow+\infty} \int \sigma: e\left(u_{h}\right) d \mu=\lim _{h \rightarrow+\infty} \int \sigma: e_{\mu}\left(u_{h}\right) d \mu \\
& =\int \sigma: e_{\mu}(u) d \mu
\end{aligned}
$$

where we have used the fact that $e_{\mu}\left(u_{h}\right)$ converges to $e_{\mu}(u)$ weakly in $L_{\mu}^{p}$ (see Remark 3.3). Therefore, since $\sigma \in \partial j_{\mu}\left(x, e_{\mu}(u)\right)$, we obtain

$$
\langle f, u\rangle=\int\left[j_{\mu}\left(x, e_{\mu}(u)\right)+j_{\mu}^{*}(x, \sigma)\right] d \mu .
$$

By Lemma 3.8 and condition iv) in (3.12), we have $j_{\mu}^{*}(x, \sigma)=1 / p^{\prime}$ so that (3.13) yields

$$
\langle f, u\rangle=\mu\left(\mathbf{R}^{n}\right) .
$$

We can repeat the computation above by using in (3.13) the Fenchel inequality instead of the equality, and we obtain

$$
\langle f, v\rangle \leq \mu\left(\mathbf{R}^{n}\right) \quad \forall v \in \operatorname{Lip}_{1, \rho}(\Omega, \Sigma) .
$$

This proves that $u$ solves problem (3.1) and therefore $\langle f, u\rangle=I(f, \Sigma, \Omega)=\mu\left(\mathbf{R}^{n}\right)$.

Let us show now that $v$ is optimal for problem (2.7). Indeed, setting $t=$ $(I / m)^{1 /(p-1)}$, we have

$\mathcal{C}(v) \leq\langle f, t u\rangle-\bar{J}(v, t u)=\langle f, t u\rangle-\int j_{v}\left(x, e_{v}(t u)\right) d v=t I-t^{p} \frac{m}{p}=\frac{I^{p^{\prime}}}{p^{\prime} m^{1 /(p-1)}}$

and so the optimality of $v$ follows from Theorem 2.3. To conclude the proof it is now enough to apply the last statement of Proposition 3.7.

\section{The scalar case}

In this section we restrict the results obtained in Sects. 2 and 3 to the scalar case. We recall that in this framework the mass optimization problem turns out to be a model for the problem of finding the best distribution $\mu$ of a given conductor in a given design region $\Omega$ in order to maximize the energy

$$
\mathcal{E}(\mu)=\inf \left\{\int j(D u) d \mu-\langle f, u\rangle: u \in \mathcal{D}\left(\mathbf{R}^{n}\right), u=0 \text { on } \Sigma\right\}
$$

among all admissible $\mu$, constrained to have a prescribed total mass, that is $\int d \mu=m$, and a support into the design region, that is spt $\mu \subset \bar{\Omega}$. Here the term $f$ represents a given heat sources density, which we assume to be a given signed measure on $\bar{\Omega}$ with finite total variation. The term scalar comes from the fact that the state variable $u$ (the temperature in the thermic model above) takes its values in $\mathbf{R}$. This allows to simplify the optimality equation (3.12) and to interpret it as the optimality condition for a Monge-Kantorovich mass transport problem. 
Consequently, we will find in some cases explicit solutions to the mass and shape optimization problems through the study of optimal transport rays.

We assume here that the design region $\Omega$ is a connected bounded open subset of $\mathbf{R}^{n}$ with a Lipschitz boundary and that $\Sigma$ is a closed subset of $\bar{\Omega}$. Moreover, for simplicity we assume that $j(z)=\frac{1}{p}|z|^{p}$ with $p>1$, which gives, with the notation of Sect. $2, \rho^{0}(z)=\rho(z)=|z|$. In this case, instead of $\operatorname{Lip}_{1, \rho}(\Omega, \Sigma)$ we simply write $\operatorname{Lip}_{1}(\Omega, \Sigma)$. We may verify that the analogous of Theorem 2.3 still holds in the scalar case, with the obvious modifications, and the quantity in (2.9) becomes

$$
\sup \left\{\langle f, u\rangle: u \in \operatorname{Lip}_{1}(\Omega, \Sigma)\right\}
$$

Therefore, a measure $\mu$ is optimal for problem (2.7) if and only if there exists a vector field $\sigma \in L_{\mu}^{\infty}\left(\bar{\Omega}, \mathbf{R}^{n}\right)$ such that $\lambda=\sigma \mu$ solves problem (2.12), which in this case takes the form

$$
I(f, \Sigma, \Omega)=\inf \left\{\int|\lambda|: \lambda \in \mathcal{M}\left(\bar{\Omega} ; \mathbf{R}^{n}\right),-\operatorname{div} \lambda=f \text { on } \mathbf{R}^{n} \backslash \Sigma\right\} .
$$

Moreover, the field $\sigma$ satisfies $|\sigma|=I(f, \Sigma, \Omega) / m$. As the optimal measure $\mu$ depends of the total mass $m$ through a multiplicative factor, we will go further assuming the normalization condition $m=I(f, \Sigma, \Omega)$.

In order to derive the Monge-Kantorovich equation we have to rephrase the construction made in Sect. 3 when considering a gradient vector operator $D u$ instead of the matrix operator $e(u)$. In this case the scheme, as depicted in [6], is simpler and

$$
X_{\mu}^{p^{\prime}}\left(\Omega ; \mathbf{R}^{n}\right)=\left\{\sigma \in L_{\mu}^{p^{\prime}}\left(\Omega ; \mathbf{R}^{n}\right): \operatorname{div}(\sigma \mu) \in \mathcal{M}\left(\mathbf{R}^{n}\right)\right\} .
$$

The set $M_{\mu}(x)$ is now a linear subspace of $\mathbf{R}^{n}$, that we call tangent space to $\mu$ at $x$ and we denote by $T_{\mu}(x)$. The tangential gradient of a smooth function $u \in \mathcal{D}\left(\mathbf{R}^{n}\right)$ is defined now through the orthogonal projector $\mathcal{P}_{\mu}(x)$ on $T_{\mu}(x)$

$$
D_{\mu} u(x)=\mathcal{P}_{\mu}(x) D u(x)
$$

and by an argument similar to the one of Lemma 3.2 we can show that the operator $D_{\mu}$ is closable. This allows us to define the space of functions with finite energy, which contains all Lipschitz functions on $\Omega$. Therefore $D_{\mu} u$ is well defined for every Lipschitz function $u$ as an element of $L_{\mu}^{\infty}\left(\Omega ; \mathbf{R}^{n}\right)$ and the optimality conditions (3.12) become as stated in [6]:

$$
\left\{\begin{aligned}
\text { i) } & -\operatorname{div}\left(D_{\mu}(u) \mu\right)=f \quad \text { on } \mathbf{R}^{n} \backslash \Sigma \\
\text { ii) } & u \in \operatorname{Lip}_{1}(\Omega, \Sigma) \\
\text { iii }) & \left|D_{\mu}(u)\right|=1 \quad \mu \text {-a.e. on } \mathbf{R}^{n} \\
\text { iv }) & \mu(\Sigma)=0 .
\end{aligned}\right.
$$

Here, due to the fact that the energy density function $j$ is isotropic, we obtain that every smooth solution $u$ of (4.1) satisfies $D_{\mu} u=D u \mu$-almost everywhere. Indeed, from ii) and iii) for $\mu$-a.e. $x$ we have $1=\left|D_{\mu} u\right| \leq|D u| \leq 1$, so that $\left|D u-D_{\mu} u\right|^{2}=|D u|^{2}-\left|D_{\mu} u\right|^{2}=0$. 
Our aim is to derive an explicit expression for solutions $\mu$ of the mass optimization problem in terms of optimal transport measures $\gamma$ associated to the Monge-Kantorovich mass transport problem. We begin by showing (this is specific to the scalar case) that the class $\operatorname{Lip}_{1}(\Omega, \Sigma)$ can be completely characterized through the geodesic semi-distance defined on $\bar{\Omega} \times \bar{\Omega}$ by

$d_{\Omega, \Sigma}(x, y)=\sup \left\{|\varphi(x)-\varphi(y)|: \varphi \in \operatorname{Lip}\left(\mathbf{R}^{n}\right),|D \varphi| \leq 1\right.$ on $\Omega, \varphi=0$ on $\left.\Sigma\right\}$.

This semi-distance will play the role of cost function in the Monge-Kantorovich mass transport problem.

Proposition 4.1. The following facts hold for the semi-distance $d_{\Omega, \Sigma}$.

i) $d_{\Omega, \Sigma}(x, y)=|x-y|$ whenever $x, y \in \Omega \backslash \Sigma$ and $|x-y|$ is small enough;

ii) $d_{\Omega, \Sigma}(x, y) \leq C|x-y|$ for all $x, y \in \bar{\Omega}$, where $C$ is a suitable constant which depends only on $\Omega$;

iii) if $\Sigma$ is empty, then $d_{\Omega, \Sigma}$ coincides with the usual geodesic distance

$$
\delta_{\Omega}(x, y)=\min \left\{\int_{0}^{1}\left|\gamma^{\prime}(t)\right| d t: \gamma \in \operatorname{Lip}([0,1] ; \bar{\Omega}), \gamma(0)=x, \gamma(1)=y\right\} ;
$$

iv) if $\Sigma$ is nonempty, then

$$
d_{\Omega, \Sigma}(x, y)=\inf \left\{\delta_{\Omega}(x, y) \wedge\left(\delta_{\Omega}\left(x, \xi_{1}\right)+\delta_{\Omega}\left(y, \xi_{2}\right)\right): \xi_{1}, \xi_{2} \in \Sigma\right\} .
$$

Proof. The only nontrivial part is the proof of iv). The inequality $\leq$ in iv) follows immediately from iii) and from the triangle inequality for $d_{\Omega, \Sigma}$. In order to prove the opposite inequality denote by $c(x, y)$ the right-hand side in iv) and, given $x, y \in \bar{\Omega}$, consider the function

$$
\varphi(z)=\frac{1}{2}\left(c(x, z)-\delta_{\Omega}(x, \Sigma)-c(y, z)+\delta_{\Omega}(y, \Sigma)\right) .
$$

It is easy to see that it satisfies $\varphi=0$ on $\Sigma,|D \varphi| \leq 1$ on $\Omega$, and $|\varphi(x)-\varphi(y)|=$ $c(x, y)$. Therefore, by the definition (4.2) we obtain $d_{\Omega, \Sigma}(x, y) \geq c(x, y)$ which concludes the proof.

Notice that the minimum in iii) is always achieved, but in general, due to the presence of the obstacle $\bar{\Omega}$, it can be nonunique, (see Example 5.7 and Fig. 6). We denote by $G\left(x_{1}, x_{2}\right)$ the set of all curves $\gamma([0,1])$ where $\gamma$ minimizes the geodesic distance $\delta_{\Omega}\left(x_{1}, x_{2}\right)$ in iii), and we call the elements of $G\left(x_{1}, x_{2}\right)$ geodesic rays. If $\Sigma$ is nonempty, we have to modify the definition of geodesic in order to fit with the semi distance $d_{\Omega, \Sigma}$. In view of iv), we denote by $D_{\Sigma}\left(x_{1}, x_{2}\right)$ the following set of two components curves:

$$
D_{\Sigma}\left(x_{1}, x_{2}\right)=\bigcup_{\left(\xi_{1}, \xi_{2}\right) \in \Sigma^{2}}\left\{S_{1} \cup S_{2}: S_{1} \in G\left(x_{1}, \xi_{1}\right), S_{2} \in G\left(x_{2}, \xi_{2}\right)\right\}
$$


and define

$$
G_{\Sigma}\left(x_{1}, x_{2}\right)= \begin{cases}G\left(x_{1}, x_{2}\right) & \text { if } d_{\Omega, \Sigma}\left(x_{1}, x_{2}\right)<\delta_{\Omega}\left(x_{1}, x_{2}\right) \\ D_{\Sigma}\left(x_{1}, x_{2}\right) & \text { if } d_{\Omega, \Sigma}\left(x_{1}, x_{2}\right)>\delta_{\Omega}\left(x_{1}, x_{2}\right), \\ G\left(x_{1}, x_{2}\right) \cup D_{\Sigma}\left(x_{1}, x_{2}\right) & \text { if } d_{\Omega, \Sigma}\left(x_{1}, x_{2}\right)=\delta_{\Omega}\left(x_{1}, x_{2}\right)\end{cases}
$$

Then by the assertion iv) of Proposition 4.1, we obtain $\mathcal{H}^{1}(S)=d_{\Omega, \Sigma}\left(x_{1}, x_{2}\right)$ and $\mathcal{H}^{1}(S \cap \Sigma)=0$ for every $S \in G_{\Sigma}\left(x_{1}, x_{2}\right)$.

Proposition 4.2. The following facts hold.

i) We have $u \in \operatorname{Lip}_{1}(\Omega, \Sigma)$ if and only if $u=0$ on $\Sigma$ and

$$
|u(x)-u(y)| \leq d_{\Omega, \Sigma}(x, y) \quad \forall x, y \in \Omega .
$$

In particular, by Proposition 4.1 ii), every function in $\operatorname{Lip}_{1}(\Omega, \Sigma)$ is Lipschitz continuous.

ii) For a function $u \in \operatorname{Lip}_{1}(\Omega, \Sigma)$ and two points $x, y \in \bar{\Omega}$, we have $\mid u(x)-$ $u(y) \mid=\delta_{\Omega, \Sigma}(x, y)$ if and only if

$$
\left|D_{S} u\right|=1 \quad \mathcal{H}^{1} \text {-a.e. on } S, \text { for every } S \in G_{\Sigma}(x, y) .
$$

iii) The multifunction $(x, y) \mapsto G_{\Sigma}(x, y)$ defined on $\bar{\Omega} \times \bar{\Omega}$ and ranging into the family of compact subsets of $\bar{\Omega}$ embedded with the Hausdorff metric topology is upper semicontinuous, hence Borel regular (we refer to the book by Castaing and Valadier [8] for all details about multifunctions and Hausdorff topology).

Proof. Let $u \in \operatorname{Lip}_{1}(\Omega, \Sigma)$ and let $\left(u_{h}\right)$ be an approximating sequence in $\mathcal{D}\left(\mathbf{R}^{n}\right)$ converging to $u$ uniformly on $\Omega$ and such that $u_{h}=0$ on $\Sigma$ and $\left|D u_{h}\right| \leq 1$ on $\Omega$. Then by the definition (4.2) of $d_{\Omega, \Sigma}$ we obtain for all $x, y \in \Omega$

$$
\left|u_{h}(x)-u_{h}(y)\right| \leq d_{\Omega, \Sigma}(x, y)
$$

and (4.4) follows as $h \rightarrow+\infty$.

Conversely, let $u$ verify (4.4) with $u=0$ on $\Sigma$. By Proposition 4.1 ii), $u$ is Lipschitz continuous on $\Omega$, and by Proposition 4.1 i), $|D u| \leq 1$ Lebesgue a.e. on $\Omega$. By a procedure similar to the one used in Lemma 3.6 we may construct a sequence $\left(u_{h}\right)$ in $\mathcal{D}\left(\mathbf{R}^{n}\right)$ which approximates $u$ uniformly, such that $u_{h}=0$ on $\Sigma$ and $\left|D u_{h}\right| \leq 1$ on $\Omega$.

To prove the second assertion, take $u \in \operatorname{Lip}_{1}(\Omega, \Sigma)$ and $x, y \in \bar{\Omega}$ such that $|u(x)-u(y)|=d_{\Omega, \Sigma}(x, y)$. Let $S \in G_{\Sigma}(x, y)$ and assume first that $S$ is a geodesic curve $\gamma$ joining $x$ to $y$, so that $d_{\Omega, \Sigma}(x, y)=\delta_{\Omega}(x, y)=\mathcal{H}^{1}(S)$. In this case, we have

$$
\begin{aligned}
|u(x)-u(y)| & =|u(\gamma(0))-u(\gamma(1))|=\left|\int_{0}^{1} D u(\gamma(t)) \cdot \gamma^{\prime}(t) d t\right| \\
& \leq \int_{S}\left|D_{S} u\right| d \mathcal{H}^{1} \leq \mathcal{H}^{1}(S)=d_{\Omega, \Sigma}(x, y) .
\end{aligned}
$$


Thus the previous inequality becomes an equality and so $\left|D_{S} u\right|=1$ must hold $\mathcal{H}^{1}$-a.e.on $S$. In the other case, $S=S_{1} \cup S_{2}$ where $S_{1}, S_{2}$ are geodesic curves joining $x$ to $x^{\prime}, y$ to $y^{\prime}$ where $x^{\prime}, y^{\prime}$ are suitable points in $\Sigma$. In the same way as above the conclusion follows from the following inequalities

$$
\begin{aligned}
|u(x)-u(y)| & \leq\left|u(x)-u\left(x^{\prime}\right)\right|+\left|u(y)-u\left(y^{\prime}\right)\right| \\
& \leq \int_{S_{1} \cup S_{2}}\left|D_{S} u\right| d \mathcal{H}^{1} \leq \mathcal{H}^{1}(S)=d_{\Omega, \Sigma}(x, y) .
\end{aligned}
$$

In order to prove assertion iii), in view of (4.3) and by the continuity of $\delta_{\Omega}-d_{\Omega, \Sigma}$, it is enough to prove separately the upper semicontinuity for the multifunctions $G$ and $D_{\Sigma}$. Let us consider a sequence $\left\{\left(x_{h}, y_{h}\right)\right\}$ converging to $(x, y)$ in $\bar{\Omega} \times \bar{\Omega}$. Let $S_{h}$ be a geodesic curve between $x_{h}$ and $y_{h}$ and assume that $\left\{S_{h}\right\}$ (or a subsequence $\left\{S_{h_{k}}\right\}$ ) converges to some $S$ in the Hausdorff convergence. By Proposition 4.1 ii), the length of $S_{h}$ is bounded uniformly by some constant $L$. Then there exists a parametrization $\gamma_{h}:[0,1] \mapsto \mathbf{R}^{n}$ of $S_{h}$ with $\gamma_{h}(0)=x_{h}$ and $\gamma_{h}(1)=y_{h}$, such that $\left|\gamma_{h}^{\prime}\right| \leq L$. Therefore, $\left\{\gamma_{h}\right\}$ is bounded in $W^{1, \infty}(0,1)$ and any uniform limit point $\gamma$ will satisfy $\gamma([0,1])=S, \gamma(0)=x, \gamma(1)=y$ and

$$
\mathcal{H}^{1}(S)=\int_{0}^{1}\left|\gamma^{\prime}\right|(t) d t \leq \liminf _{h \rightarrow \infty} \int_{0}^{1}\left|\gamma_{h}^{\prime}\right|(t) d t \leq \limsup _{h \rightarrow \infty} \delta_{\Omega}\left(x_{h}, y_{h}\right)=\delta_{\Omega}(x, y) .
$$

Thus $S$ belongs to $G(x, y)$ and the upper semicontinuity property of $G$ is proved. The case of $D_{\Sigma}$ can be treated in a similar way by considering $S_{h}=S_{1, h} \cup S_{2, h}$ where $S_{1, h}$ (respectively $S_{2, h}$ ) is a geodesic curve joining $x_{h}$ to $\xi_{1, h}$ (respectively $y_{h}$ to $\left.\xi_{2, h}\right)$ with $\left(\xi_{1, h}, \xi_{2, h}\right) \in \Sigma^{2}$. Then, as $\Sigma$ is compact, we may assume, possibly passing to subsequences, that $\left(\xi_{1, h}, \xi_{2, h}\right)$ converges to some $\left(\xi_{1}, \xi_{2}\right)$ and that $\left(S_{1, h}, S_{2, h}\right)$ converges to a pair of curves $\left(S_{1}, S_{2}\right)$ joining $x$ to $\xi_{1}$ and $y$ to $\xi_{2}$. Then we apply the previous step to conclude that each $S_{i}$ is a geodesic curve, so that $S_{1} \cup S_{2}$ belongs to $D_{\Sigma}(x, y)$.

Let us now consider the mass transport problem associated to the cost function $d_{\Omega, \Sigma}$. Given two measures $\lambda_{1}, \lambda_{2} \in \mathcal{M}^{+}(\bar{\Omega})$ such that $\lambda_{1}(\bar{\Omega})=\lambda_{2}(\bar{\Omega})$ we define the distance

$$
\Phi\left(\lambda_{1}, \lambda_{2}\right)=\min \left\{\int d_{\Omega, \Sigma}(x, y) \gamma(d x, d y): \gamma \in \Gamma\left(\lambda_{1}, \lambda_{2}\right)\right\}
$$

where $\Gamma\left(\lambda_{1}, \lambda_{2}\right)$ is the class of measures of $\mathcal{M}^{+}(\bar{\Omega} \times \bar{\Omega})$ whose marginals are $\lambda_{1}$ and $\lambda_{2}$, i.e.

$$
\lambda_{1}(B)=\gamma(B \times \bar{\Omega}) \quad \text { and } \quad \lambda_{2}(B)=\gamma(\bar{\Omega} \times B) \quad \text { for every Borel set } B \subset \bar{\Omega} \text {. }
$$

Remark 4.3. By the continuity of $d_{\Omega, \Sigma}$ on the compact set $\bar{\Omega} \times \bar{\Omega}$, we find easily that the minimum in (4.5) is achieved. Moreover, thanks to the first assertion of Theorem 4.5 below, it turns out that $\Phi$ defines a homogeneous semidistance on probability measures on $\bar{\Omega}$ : indeed

$$
\Phi\left(\lambda_{1}, \lambda_{2}\right)=\sup \left\{\int \varphi d \lambda_{1}-\int \varphi d \lambda_{2}: \varphi \in \operatorname{Lip}_{1}(\Omega, \Sigma)\right\}
$$


is a 1-homogeneous subadditive function of $\lambda_{1}-\lambda_{2}$ vanishing if and only if the support of $\lambda_{1}-\lambda_{2}$ is contained in $\Sigma$. We may extend $\Phi$ by setting $\Phi\left(\lambda_{1}, \lambda_{2}\right)=+\infty$ if $\lambda_{1}(\bar{\Omega}) \neq \lambda_{2}(\bar{\Omega})$.

Remark 4.4. In the case $\Sigma=\varnothing$ and $\Omega=\mathbf{R}^{n}$ the function $\Phi$ in (4.5) is the Kantorovich distance between $\lambda_{1}$ and $\lambda_{2}$. A celebrated result is that in this case the distance $\Phi\left(\lambda_{1}, \lambda_{2}\right)$ can be expressed by

$$
\inf \left\{\int|x-T(x)| d \lambda_{1}(x): T^{\#}\left(\lambda_{1}\right)=\lambda_{2}\right\}
$$

whenever this last is finite, where the infimum is taken over all transport mappings $T: \mathbf{R}^{n} \rightarrow \mathbf{R}^{n}$ and $T^{\#}$ is the push-forward operator. For the conditions on $\lambda_{1}$ and $\lambda_{2}$ which imply the existence of an optimal transport map $T$ in (4.6) we refer to the recent books $[14,23]$. For variants of this result in case of different cost functionals appearing in (4.6) we refer to [16].

The next result makes the link between the semidistance $\Phi$ and the quantity $I(f, \Sigma, \Omega)$ defined in (2.9) which is directly related to the Monge-Kantorovich problem of Sect. 3 .

\section{Theorem 4.5. The following facts hold.}

i) If two measures $\lambda_{1}, \lambda_{2} \in \mathcal{M}^{+}(\bar{\Omega})$ are such that $\lambda_{1}(\bar{\Omega})=\lambda_{2}(\bar{\Omega})$, then setting $f=\lambda_{1}-\lambda_{2}$ we have

$$
\Phi\left(\lambda_{1}, \lambda_{2}\right)=\Phi\left(f^{+}, f^{-}\right)=I(f, \Sigma, \Omega) .
$$

ii) Let $f \in \mathcal{M}(\bar{\Omega})$ and let $c=\int d f$. We denote by $v$ any probability measure on $\Sigma$ in case it is not empty. We have

$$
\begin{aligned}
I(f, \Sigma, \Omega) & =\inf \left\{\Phi\left(\lambda_{1}, \lambda_{2}\right): \lambda_{2}-\lambda_{1}=f \text { on } \bar{\Omega} \backslash \Sigma\right\} \\
& = \begin{cases}\Phi\left(f^{+}, f^{-}\right) & \text {if } \Sigma=\emptyset(+\infty \text { if } c \neq 0) \\
\Phi\left(f^{+}, f^{-}+c \nu\right) & \text { if } \Sigma \neq \emptyset \text { and } c \geq 0 \\
\Phi\left(f^{+}-c \nu, f^{-}\right) & \text {if } \Sigma \neq \emptyset \text { and } c \leq 0 .\end{cases}
\end{aligned}
$$

Proof. First we notice that, for elements of $\mathcal{M}^{+}(\bar{\Omega})$ such that $\lambda_{1}(\bar{\Omega})=\lambda_{2}(\bar{\Omega})$, $\lambda_{1}^{\prime}(\bar{\Omega})=\lambda_{2}^{\prime}(\bar{\Omega})$, we have

$$
\Phi\left(\lambda_{1}, \lambda_{2}\right)=\Phi\left(\lambda_{1}^{\prime}, \lambda_{2}^{\prime}\right) \quad \text { whenever } \lambda_{1}^{\prime}-\lambda_{2}^{\prime}=\lambda_{1}-\lambda_{2} \text { on } \bar{\Omega} \backslash \Sigma .
$$

Indeed, as $d_{\Omega, \Sigma}$ vanishes on $\Sigma \times \Sigma$ and on the diagonal, without changing the cost functional, we may add (or subtract) to every competitor $\gamma$ in (4.5) the measure $\delta$ given by

$$
\langle\delta, \varphi\rangle:=\int_{\bar{\Omega} \backslash \Sigma} \varphi(x, x) v_{i}(d x)+\frac{1}{m} \int_{\Sigma \times \Sigma} \varphi(x, y) v_{1} \otimes v_{2}(d x, d y)
$$

where $v_{i}:=\lambda_{i}^{\prime}-\lambda_{i}$ and $m:=\int_{\Sigma} v_{i}(i=1,2)$, without changing the cost functional. 
Then the first equality of $\mathrm{i}$ ) is deduced from (4.7) by taking $\lambda_{1}^{\prime}=f^{+}, \lambda_{2}^{\prime}=f^{-}$. In fact, if $\Sigma$ is nonempty, we may start with a non balanced signed measure $f$ $\left(c=\int d f\right)$, and replace in (4.7) $\lambda_{1}^{\prime}$ by $f^{-}-c v$ if $c<0$ (respectively $\lambda_{2}^{\prime}$ by $f^{-}+c v$ if $c>0$ ) where $v$ is a probability measure on $\Sigma$. In this case, we obtain

$$
\Phi\left(\lambda_{1}, \lambda_{2}\right)= \begin{cases}\Phi\left(f^{+}, f^{-}+c \nu\right) & \text { if } c>0 \\ \Phi\left(f^{+}-c v, f^{-}\right) & \text {if } c<0\end{cases}
$$

whenever $\lambda_{2}-\lambda_{1}=f$ on $\bar{\Omega} \backslash \Sigma$. Hence the second equality of i) and ii) are consequences of the following claim: for every signed measure $f$ on $\bar{\Omega}$

$$
I(f, \Sigma, \Omega)=\inf \left\{\Phi\left(\lambda_{1}, \lambda_{2}\right): \lambda_{2}-\lambda_{1}=f \text { on } \bar{\Omega} \backslash \Sigma\right\} .
$$

We introduce the following function $G$ defined on $\mathcal{C}(\bar{\Omega} \times \bar{\Omega})$ :

$$
G(p):=\inf _{\varphi \in \mathcal{C}(\bar{\Omega})}\left\{\begin{array}{l}
-\langle f, \varphi\rangle: \varphi=0 \text { on } \Sigma, \\
\varphi(y)-\varphi(x)+p(x, y) \leq d_{\Omega, \Sigma}(x, y) \text { on } \Omega \times \Omega
\end{array}\right\} .
$$

It turns out that $G$ is convex and, by the characterization of $\operatorname{Lip}_{1}(\Omega, \Sigma)$ given in Proposition 4.2, we have $G(0)=-I(f, \Sigma, \Omega)$ (here $p$ plays the role of a perturbation parameter). Let us compute the Moreau-Fenchel conjugate of $G$ in the duality $(\mathcal{M}(\bar{\Omega} \times \bar{\Omega}), \mathcal{C}(\bar{\Omega} \times \bar{\Omega}))$. Given $\gamma \in \mathcal{M}(\bar{\Omega} \times \bar{\Omega})$ whose marginals are denoted by $\lambda_{1}, \lambda_{2}$, we have

$$
\begin{aligned}
G^{*}(\gamma) & =\sup \left\{\int p d \gamma-G(p): p \in \mathcal{C}(\bar{\Omega} \times \bar{\Omega})\right\} \\
& =\sup \left\{\begin{array}{ll}
\int \bar{\Omega} \times \bar{\Omega} \\
\varphi(y)-\varphi(x)+p(x, y) \leq d_{\Omega, \Sigma}(x, y) \text { on } \Omega^{2}
\end{array}\right\} \\
& = \begin{cases}\int_{\bar{\Omega} \times \bar{\Omega}} d_{\Omega, \Sigma}(x, y) d \gamma+\sup _{\varphi=0} \text { on } \Sigma\left\{\int_{\bar{\Omega} \times \bar{\Omega}}(\varphi(x)-\varphi(y)) d \gamma+\int_{\bar{\Omega}} \varphi d f\right\} \\
+\infty & \text { if } \gamma \geq 0 \\
\text { otherwise }\end{cases} \\
= & \begin{cases}\int_{\bar{\Omega} \times \bar{\Omega}} d_{\Omega, \Sigma}(x, y) d \gamma & \text { if } \gamma \geq 0 \text { and } \lambda_{2}-\lambda_{1}=f \text { on } \bar{\Omega} \backslash \Sigma \\
+\infty & \text { otherwise. }\end{cases}
\end{aligned}
$$

Thus claim (4.8) amounts to showing that $G(0)=-\inf G^{*}=G^{* *}(0)$. The inequality $G^{* *}(0) \leq G(0)$ being always true, we have to prove that $G(0) \leq G^{* *}(0)$. We may assume that $G(0)>-\infty$ (i.e. $I(f, \Sigma, \Omega)<+\infty)$. As $G$ is convex and finite at 0 , the claim (4.8) will be a consequence of the lower semicontinuity of $G$ at 0 . Let $\left\{p_{h}\right\}_{h \in \mathbf{N}}$ be a sequence in $\mathcal{C}(\bar{\Omega} \times \bar{\Omega})$ such that $p_{h}$ converge uniformly to 0 . Then there exists a sequence $\left\{\varphi_{h}\right\}$ in $\mathcal{C}(\bar{\Omega})$ such that

$$
\begin{aligned}
& G\left(p_{h}\right)>-\int_{\bar{\Omega}} \varphi_{h} d f-\frac{1}{h}, \quad \varphi_{h}=0 \text { on } \Sigma, \\
& \varphi_{h}(y)-\varphi_{h}(x)+p_{h}(x, y) \leq d_{\Omega, \Sigma}(x, y) \text { on } \Omega^{2} .
\end{aligned}
$$


Then by the assertion ii) of Proposition 4.1, there holds for every $(x, y) \in \Omega^{2}$ and for a suitable constant $C$ :

$$
\left|\varphi_{h}(x)-\varphi_{h}(y)\right| \leq C|x-y|+\left\|p_{h}\right\|_{\infty} .
$$

As $\left\|p_{h}\right\|_{\infty}$ tends to $0,(4.10)$ implies that the sequence $\left\{\varphi_{h}\right\}$ is equicontinuous on $\bar{\Omega}$. Then, setting $c_{h}:=\varphi_{h}\left(x_{0}\right)$ for a fixed $x_{0} \in \bar{\Omega}$, we deduce the boundedness of the set $\left\{\varphi_{h}(x)-c_{h}\right\}$ for every $x \in \bar{\Omega}$. By Ascoli-Arzelà's theorem, the set $\left\{\varphi_{h}-c_{h}: h \in \mathbf{N}\right\}$ is precompact in $\mathcal{C}(\bar{\Omega})$.

In the case $\Sigma \neq \emptyset$, we may take $x_{0} \in \Sigma$; then $c_{h}=0$ and by (4.9) any cluster point $\varphi$ of $\left\{\varphi_{h}: h \in \mathbf{N}\right\}$ satisfies $\varphi=0$ on $\Sigma$ and $\varphi(y)-\varphi(x) \leq d_{\Omega, \Sigma}(x, y)$ on $\Omega^{2}$. Thus, for a suitable subsequence $h_{k}$, we obtain

$$
\liminf _{h} G\left(p_{h}\right)=\lim _{k} G\left(p_{h_{k}}\right) \geq \liminf _{k} \int_{\bar{\Omega}}-\varphi_{h_{k}} d f \geq-\int_{\bar{\Omega}} \varphi d f \geq G(0) .
$$

The case $\Sigma=\emptyset$, can be concluded in the same way noticing that, by the assumption $-G(0)=I(f, \Sigma, \Omega)<+\infty$, the measure $f$ has average zero and then $\int\left(\varphi_{h}-c_{h}\right) d f=\int \varphi_{h}$ for every $h$.

We are now able to reconstruct optimal mass distributions $\mu$ from the optimal transport measures $\gamma$ for (4.5). This means that the optimal $\mu$ is fibrated by the subset of geodesic along which the transport takes place.

Theorem 4.6. Let $f \in \mathcal{M}(\bar{\Omega})$ and let $\gamma$ be a solution of (4.5) being $\left(\lambda_{1}, \lambda_{2}\right)$ any pair such that $\lambda_{2}-\lambda_{1}=f$ on $\bar{\Omega} \backslash \Sigma$. Then for every Borel selection $(x, y) \mapsto S_{x y}$ of the multifunction $G_{\Sigma}$, the measure $\mu$ defined by

$$
\langle\mu, \varphi\rangle=\int_{\bar{\Omega} \times \bar{\Omega}}\left(\int_{S_{x y}} \varphi d \mathcal{H}^{1}\right) d \gamma(d x, d y), \quad \varphi \in \mathcal{C}(\bar{\Omega})
$$

is optimal for problem (2.7) with $m=I(f, \Sigma, \Omega)$. Moreover, denoting by $\tau_{S_{x y}}$ the unit tangent vector to $S_{x y}$ (oriented from $x$ to $y$ ), the field $\sigma:=D_{\mu} u$ given in (4.1) can be represented as the Radon-Nikodym derivative with respect to $\mu$ of the vector measure

$$
\langle\sigma \mu, \Psi\rangle:=\int_{\bar{\Omega} \times \bar{\Omega}}\left(\int_{S_{x y}} \Psi \cdot \tau_{S_{x y}} d \mathcal{H}^{1}\right) d \gamma(d x, d y), \quad \psi \in \mathcal{C}\left(\bar{\Omega} ; \mathbf{R}^{n}\right) .
$$

Proof. As all curves $S \in G_{\Sigma}(x, y)$ lie in $\bar{\Omega}$ and satisfy $\mathcal{H}^{1}(S \cap \Sigma)=0$, it is clear that the measure $\mu$ defined by (4.11) is supported in $\bar{\Omega}$ and that $\mu(\Sigma)=0$. According to our observations preceding (4.1), we may assume that $I=I(f, \Sigma, \Omega)=m$. Then, since the density $\sigma$ given by (4.12) satisfies $|\sigma|=1 \mu$-a.e., we have by (4.11) and Proposition (4.2)

$$
\begin{aligned}
\int_{\bar{\Omega}}|\sigma| d \mu & =\int_{\bar{\Omega}} \mu=\int_{\bar{\Omega} \times \bar{\Omega}} \mathcal{H}^{1}\left(S_{x y}\right) \gamma(d x, d y) \\
& =\int_{\bar{\Omega} \times \bar{\Omega}} d_{\Omega, \Sigma}(x, y) \gamma(d x, d y)=I(f, \Sigma, \Omega)=m .
\end{aligned}
$$


Therefore $\lambda:=\sigma \mu$ solves (2.12) provided $\sigma$ satisfies the condition $-\operatorname{div} \sigma \mu=f$ on $\mathbf{R}^{n} \backslash \Sigma$. Let us apply (4.12) to $\Psi=D \varphi$ where $\varphi$ is a test function in $\mathcal{D}\left(\mathbf{R}^{n}\right)$. We obtain

$$
\begin{aligned}
\langle-\operatorname{div} \sigma \mu, \varphi\rangle & =\int_{\bar{\Omega} \times \bar{\Omega}}\left(\int_{S_{x y}} D_{\tau_{x y}} \varphi d \mathcal{H}^{1}\right) \gamma(d x, d y) \\
& =\int_{\bar{\Omega} \times \bar{\Omega}}(\varphi(y)-\varphi(x)) \gamma(d x, d y) \\
& =\left\langle\lambda_{2}-\lambda_{1}, \varphi\right\rangle .
\end{aligned}
$$

Thus the measure $-\operatorname{div} \sigma \mu=\lambda_{2}-\lambda_{1}$ agrees with $f$ on $\mathbf{R}^{n} \backslash \Sigma$. The optimality of $\mu$ follows by applying to $\lambda:=\sigma \mu$ the assertion iii) of Theorem 3.3.

Remark 4.7. A meaningful consequence of this result is that there exist optimal measures $\mu$ supported by the geodesic hull of $K:=\operatorname{spt} f \cup \Sigma$, that is by the set $\cup\left\{G_{\Sigma}(x, y):(x, y) \in K \times K\right\}$. An interesting question is the validity of the converse implication: does the optimality of $\mu$ imply its representation under the form (4.11), being $\gamma$ an optimal transport measure? This question has been solved in [14] in the case of a Lipschitz source term $f$ verifying the condition spt $f^{+} \cap \operatorname{spt} f^{-}=\emptyset, \Omega=\mathbf{R}^{n}$ and $\Sigma=\emptyset$ (then $K$ is the convex hull of spt $f$ ), by using a quite involved approximation procedure and by solving an ODE along geodesic rays.

\section{Some examples}

In this section we present some examples of optimal structures both in the scalar case as well as in the case of elasticity. The optimality of the described structures will be tested through the Monge-Kantorovich conditions (3.12).

Example 5.1. We start with the following problem in elasticity: distribute in $\mathbf{R}^{2}$ a given amount of mass in order to minimize the elastic compliance related to the force field $f=\delta_{A} \tau_{1}+\delta_{B} \tau_{2}+\delta_{C} \tau_{3}$ described in figure below.

$$
{ }_{\mathrm{C}} \uparrow^{\tau_{3}}
$$

O.

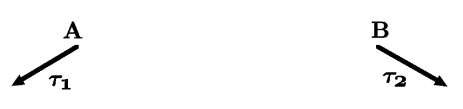

Fig. 1. The force $f$ 
A first guess for the optimal measure $\mu$, when we deal with the usual stored energies of the linear isotropic elasticity, could be choosing any of the two onedimensional structures of figure below, where the total mass is prescribed, and the one-dimensional density of $\mu$ on bars is constant.
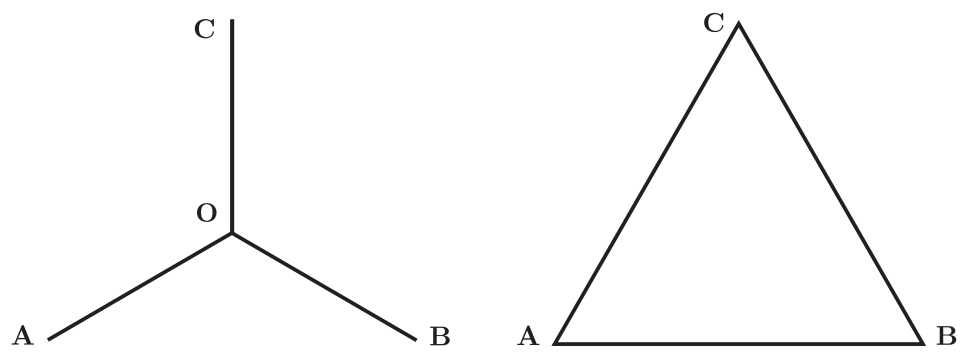

Fig. 2. Two structures that are not optimal

By the results of Sect. 3 we know that a mass distribution $\mu$ is optimal if and only if a multiple of it satisfies the Monge-Kantorovich equation (3.12) which reads in this case:

$$
\left\{\begin{aligned}
\text { i) } & -\operatorname{div}(\sigma \mu)=f \quad \text { on } \mathbf{R}^{2} \\
\text { ii) } & \sigma \in \partial j_{\mu}\left(x, e_{\mu}(u)\right) \quad \mu \text {-a.e. on } \mathbf{R}^{2} \\
\text { iii) } & u \in \operatorname{Lip}_{1, \rho}\left(\mathbf{R}^{2}\right) \\
\text { iv) } & j_{\mu}\left(x, e_{\mu}(u)\right)=1 / p \quad \mu \text {-a.e. on } \mathbf{R}^{2} .
\end{aligned}\right.
$$

Let us consider the case of a linear isotropic stored energy

$$
j(z)=\frac{\alpha}{2}\left|\operatorname{tr}\left(z^{s y m}\right)\right|^{2}+\beta\left|z^{s y m}\right|^{2}
$$

where $\alpha$ and $\beta$ are the Lamé constants in dimension two. A straightforward calculation shows that for a given total mass $m$ the compliances of the two structures $\bar{\mu}_{1}$ and $\bar{\mu}_{1}$ of Fig. 2 coincide; we shall now prove that none of them is optimal, that is

$$
\inf \left\{\mathcal{C}(\mu): \mu \in \mathcal{M}^{+}\left(\mathbf{R}^{2}\right), \int d \mu=m\right\}<\mathcal{C}\left(\bar{\mu}_{1}\right)=\mathcal{C}\left(\bar{\mu}_{2}\right) .
$$

By rescaling the mass we may take for instance the case $\mu=\bar{\mu}_{1}=\mathcal{H}^{1}\llcorner S$ and we argue by contradiction that is we assume the existence of some $u \in \operatorname{Lip}_{1, \rho}\left(\mathbf{R}^{2}\right)$ such that $(u, \mu)$ satisfies (5.1). Equations i), ii), iv) determine the tangent component of $u$ on $S=O A \cup O B \cup O C$ up to a constant that we fix in order to have $u(0)=0$. We have $j_{\mu}\left(e_{\mu}(u)\right)=1 / 2 \mathcal{H}^{1}$-a.e. on $S$, and setting $c=2 j_{\mu}\left(\tau_{i} \otimes \tau_{i}\right)$ (which by isotropy does not depend on the index $i$ ), we deduce

$$
j^{*}(\eta \otimes \eta)=\frac{1}{2 c} \quad \text { for every unit vector } \eta \text {. }
$$


Moreover, we obtain for the tangential component $v(s)=u\left(s \tau_{i}\right) \cdot \tau_{i}$ on $S$

$$
\frac{1}{2} j_{\mu}\left(\tau_{i} \otimes \tau_{i} v^{\prime}\right)=\left|v^{\prime}\right|^{2} \frac{c}{2}
$$

which gives

$$
v(s)=s c^{-1 / 2} .
$$

Since $u$ is defined on the whole $\mathbf{R}^{2}$ and satisfies (5.1) iii) by applying Lemma 3.6 with the two dimensional Lebesgue measure,we must have

$$
j(e(u)) \leq 1 / 2 \quad \text { a.e. on } \mathbf{R}^{2} .
$$

Let us now consider the matrix

$$
\sigma_{0}(x)= \begin{cases}e_{1} \otimes e_{1} & \text { on triangle } A O B \\ e_{+} \otimes e_{+} & \text {on triangle } A O C \\ e_{-} \otimes e_{-} & \text {on triangle } C O B\end{cases}
$$

where $e_{1}=A B /|A B|, e_{+}=A C /|A C|, e_{-}=B C /|B C|$. We have for every $t \in \mathbf{R}$

$$
1 / 2 \geq j(e(u)) \geq t \sigma_{0}: e(u)-t^{2} j^{*}\left(\sigma_{0}\right)=t \sigma_{0}: e(u)-\frac{t^{2}}{2 c}
$$

where we have used (5.3) in the last equality. By integration, denoting by $\Delta$ the triangle $A B C$, by $n(x)$ a unit vector normal to $S$, and to $\partial \Delta$, and by [.] the jump across $S$, we obtain

$$
\begin{aligned}
\frac{|\Delta|}{2} & \geq t \int_{\Delta} \sigma_{0}: e(u) d x-\frac{t^{2}|\Delta|}{2 c} \\
& =t \int_{S}\left[\sigma_{0} n\right] \cdot u d \mathcal{H}^{1}+t \int_{\partial \Delta}\left(\sigma_{0} n\right) \cdot u d \mathcal{H}^{1}-\frac{t^{2}|\Delta|}{2 c} .
\end{aligned}
$$

By construction $\sigma_{0} n=0$ on $\partial \Delta$, and the jump $\left[\sigma_{0} n\right]$ is purely tangential:

$$
\left[\sigma_{0} n\right]=\frac{\sqrt{3}}{2} \tau_{i} \quad \text { on } S_{i}
$$

Thus, (5.4) and (5.5) yield

$$
1 \geq \frac{t \sqrt{3}}{|\Delta| \sqrt{c}} \int_{S}|x| d \mathcal{H}^{1}-\frac{t^{2}}{c}=\frac{t \sqrt{3}}{|\Delta| \sqrt{c}} \frac{|S|^{2}}{6}-\frac{t^{2}}{c}
$$

and so, taking the supremum with respect to $t$,

$$
1 \geq \frac{1}{48} \frac{|S|^{4}}{|\Delta|^{2}}
$$

Since $12|\Delta|=\sqrt{3}|S|^{2}$ the last inequality is an equality, which implies that all previous inequalities are actually equalities. In particular we obtain for a suitable $t \in \mathbf{R}$

$$
j(e(u))+j^{*}\left(t \sigma_{0}\right)=t \sigma_{0}: e(u)
$$


which gives

$$
e(u)=\partial j^{*}\left(t \sigma_{0}\right)=\frac{t}{2 \beta} \sigma_{0}-\frac{t \alpha}{4 \beta(\alpha+\beta)} I .
$$

From this last relation we deduce that the three matrices $e_{1} \otimes e_{1}, e_{+} \otimes e_{+}, e_{-} \otimes e_{-}$ must be rank one connected and have to satisfy the equalities

$$
\left\{\begin{array}{l}
\left(e_{1} \otimes e_{1}\right) \tau_{1}=\left(e_{+} \otimes e_{+}\right) \tau_{1} \\
\left(e_{1} \otimes e_{1}\right) \tau_{2}=\left(e_{-} \otimes e_{-}\right) \tau_{2} \\
\left(e_{+} \otimes e_{+}\right) \tau_{3}=\left(e_{-} \otimes e_{-}\right) \tau_{3}
\end{array}\right.
$$

which is impossible.

Remark 5.2. By repeating the argument above for a stored energy density $j(z)$ which is $p$-homogeneous, isotropic and convex, we can show that if the structures of Fig. 2 are optimal, then there exist three matrices $A_{1} \in \partial j^{*}\left(e_{1} \otimes e_{1}\right), A_{+} \in \partial j^{*}\left(e_{+} \otimes e_{+}\right)$, $A_{-} \in \partial j^{*}\left(e_{-} \otimes e_{-}\right)$such that

$$
\left\{\begin{array}{l}
A_{1} \tau_{1}=A_{+} \tau_{1} \\
A_{1} \tau_{2}=A_{-} \tau_{2} \\
A_{+} \tau_{3}=A_{-} \tau_{3} .
\end{array}\right.
$$

Remark 5.3. If we consider the so called two-dimensional Michell energy density

$$
j_{0}(z)=\frac{1}{2}\left|\left\|z^{s y m} \mid\right\|^{2}\right.
$$

where ||$|\cdot|||$ denotes the operator norm on symmetric matrices, the corresponding stress potential is given by

$$
j_{0}^{*}(\sigma)=\frac{1}{2}\left(\left|t_{1}\right|+\left|t_{2}\right|\right)^{2}
$$

where $t_{1}$ and $t_{2}$ denote the eigenvalues of the symmetric matrix $\sigma$. Then we have for every unit vector $\xi$

$$
j_{0}(I)+j_{0}^{*}(\xi \otimes \xi)=1
$$

that is $I \in \partial j_{0}^{*}(\xi \otimes \xi)$ for every $\xi \in \mathbf{R}^{2}$. Therefore the structures of Fig. 2 solve the Monge-Kantorovich equation (5.1) with $u(x)=x$, hence they are optimal.

Remark 5.4. The structures of Fig. 2 turn out to be optimal among all onedimensional structures; indeed for a one-dimensional structure $\mu$ the two energies $j$ and $j_{0}$ of (5.2) and (5.7) coincide and so, by Remark 5.3 the optimum is reached on the ones of Fig. 2. As a consequence, we can assert that for the case (5.2) of linear elasticity, no one-dimensional structure gives the optimum. A numerical computation by F. Golay and Seppecher (see [18]) shows for the case (5.2) the following optimal mass distribution. 


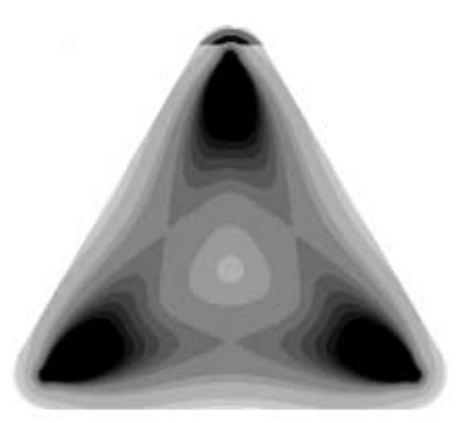

Fig. 3. The two-dimensional optimal mass distribution

We consider now some examples for optimal conductivity problems where the state function $u$ is scalar, and so the Monge-Kantorovich conditions (3.12) reduce to (4.1). In each of the following three examples we shall specify the optimal transport measure $\gamma$ and the optimal mass distribution $\mu$ given by (4.11).

Example 5.5. Let us consider a continuous plane curve $S$ in polar coordinates, $r=h(\theta)$, with length $L$, and let $f$ be the heat sources density made by a onedimensional constant density on the curve $S$ and a point concentration at the origin, that is

$$
f=\mathcal{H}^{1}\left\llcorner S-L \delta_{O} .\right.
$$

Then, the unique admissible transport $\gamma$ is given by

$$
\gamma=\mathcal{H}^{1}\left\llcorner S \otimes L \delta_{O}\right.
$$

so that the optimal pair $\left(\mu_{0}, u_{0}\right)$ is given by

$$
\mu_{0}=\frac{c}{r} \sqrt{h^{2}(\theta)+\left|h^{\prime}(\theta)\right|^{2}} \mathcal{H}^{2}\left\llcorner R, \quad u_{0}=\frac{r}{c}\right.
$$

for a suitable constant $c>0$, where $R$ is the set $0 \leq r \leq h(\theta)$. In case $h$ is a $B V$ function presenting a jump $\left[h^{-}, h^{+}\right]$at some $\theta_{0}$, then the additional concentration $c\left(h^{+}-r \vee h^{-}\right) \mathcal{H}^{1}$ occurs on the corresponding ray, which shows that optimal measures may have terms of lower dimension. In Fig. 4 we display the optimal density $\mu_{0}$ for a particular plane curve.

Example 5.6. We consider now the case of a rectangle $R=[0, L] \times[0,1]$ in $\mathbf{R}^{2}$, we take the Dirichlet region $\Sigma=[L / 3,2 L / 3] \times\{1\}$ and the source term $f$ given by the one-dimensional densities 2 on the left side and -1 on the right side, that is

$$
f=2 \mathcal{H}^{1}\left\llcorner(\{0\} \times[0,1])-\mathcal{H}^{1}\llcorner(\{L\} \times[0,1]) .\right.
$$

Note that in this example the average of $f$ is not zero, so that some measure on $\Sigma$ must be considered in the mass transport problem as a compensation term. The 


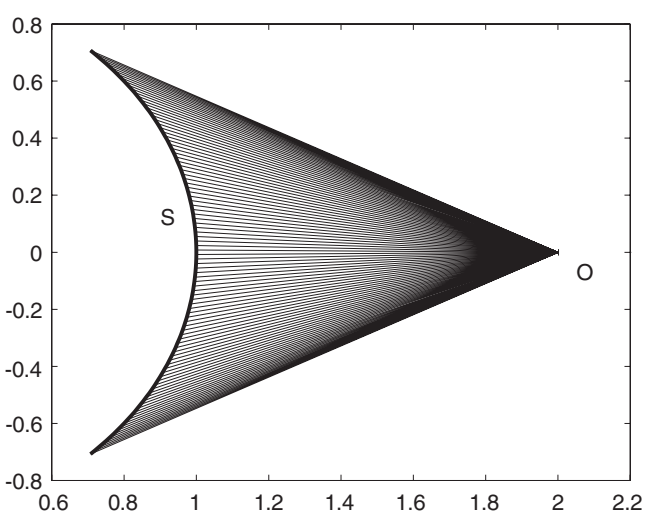

Fig. 4. The optimal mass distribution

problem specified in Theorem 4.5 is solved if we take, with the same notation used there:

$$
\begin{aligned}
\gamma= & 2 \mathcal{H}^{1}\left\llcorner( \{ 0 \} \times [ 0 , x _ { 0 } ] ) \otimes \mathcal { H } ^ { 1 } \left\llcorner\left(\{L\} \times\left[0,2 x_{0}\right]\right)\right.\right. \\
& +2 \mathcal{H}^{1}\left\llcorner\left(\{0\} \times\left[x_{0}, 1\right]\right) \otimes k_{A} \delta_{A}+k_{B} \delta_{B} \otimes \mathcal{H}^{1}\left\llcorner\left(\{L\} \times\left[2 x_{0}, 1\right]\right)\right.\right.
\end{aligned}
$$

where $A=(L / 3,1), B=(2 L / 3,1), k_{A}=2\left(1-x_{0}\right), k_{B}=1-2 x_{0}$. Here the point $x_{0}$ depends on $L$ according to the formula

$$
x_{0}=\left(\frac{9-\sqrt{9+28 L^{2}}}{12}\right)^{+} .
$$

The optimal measure $\mu$ is then deduced from $\gamma$ through (4.11). Figure 5 below represents the optimal measure $\mu$ in the case $L=1$.

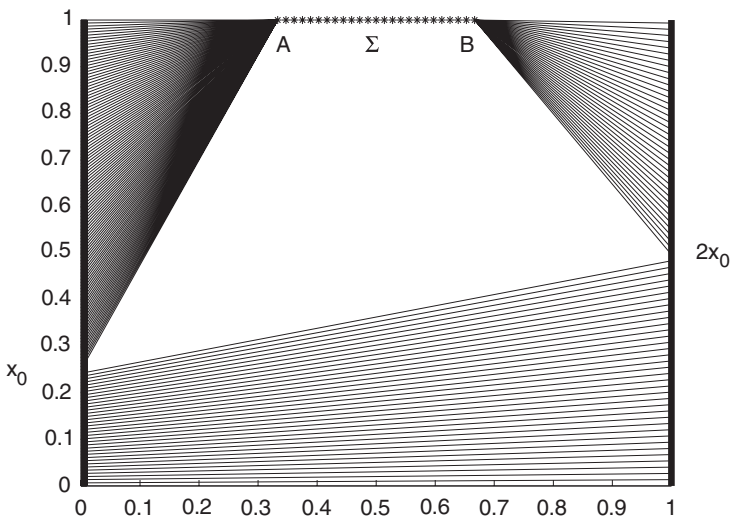

Fig. 5. The optimal mass distribution for $L=1$ 
Example 5.7. We consider now a case when the design region is not convex; the geodesic rays will then no longer be unique and rectilinear. We take for $\Omega$ the complement of the unit disk in $\mathbf{R}^{2}$ and we take for the source term a onedimensional constant density on the segment $S=\{(-2, t):|t| \leq 1\}$ and a point concentration at the point $A=(1,0)$, that is

$$
f=\mathcal{H}^{1}\left\llcorner S-2 \delta_{A}\right. \text {. }
$$

The optimal transport measure $\gamma$ is in this case simply

$$
\gamma=\mathcal{H}^{1}\left\llcorner S \otimes 2 \delta_{A}\right.
$$

but, due to the nonconvexity of $\Omega$, and hence to the presence of an obstacle for geodesic rays, the optimal mass distribution $\mu$ is of the form

$$
\mu=\alpha(x) \mathcal{H}^{2}\left\llcorner\Omega+\beta(x) \mathcal{H}^{1}\llcorner\partial \Omega\right.
$$

for suitable densities $\alpha(x)$ and $\beta(x)$. Figure 6 below gives a representation of the optimal measure $\mu$.

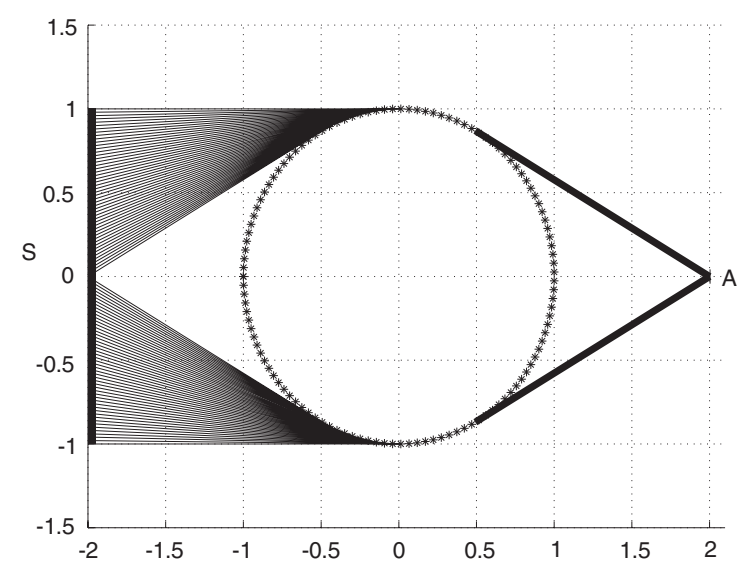

Fig. 6. The optimal mass distribution with an obstacle

We conclude this section by addressing some perspectives and open questions that seem to us challenging.

In the scalar case we have seen in Remark 4.7 that there are (actually we believe it is true for all) optimal measures supported by the geodesic hull of spt $f \cup \Sigma$ with respect to the semi-distance $d_{\Omega, \Sigma}$. In particular, in the case where the design region $\Omega$ coincides with $\mathbf{R}^{n}$, if spt $f$ is bounded we obtain that spt $\mu_{\text {opt }}$ is bounded as well. This last issue seems to be reasonable to occur also in the case of elasticity, and numerical computations support this conjecture. However, up to now we have been unable to prove this fact, even in the particular case of Example 5.1.

Another question is related to the behaviour of shape optimization problems for linear elasticity, where we restrict the class of admissible measures to the ones of 
the form $1_{\omega} d x$ with $m=\operatorname{meas}(\omega)$ a small parameter. The question is to determine the limit problem as $m \rightarrow 0$, in the sense of $\Gamma$-convergence, for the rescaled compliances

$$
\mathcal{C}_{m}(\mu)=m \mathcal{C}(m \mu) \quad\left(\text { being } \mu=\frac{1}{m} 1_{\omega} d x\right) .
$$

The form of the limit problem has only been conjectured in [1] in the cases $n=2$ and $n=3$ for the linear isotropic elasticity, and is related to the Michell problem in the case $n=2$.

A final question is concerned with a deep difference between the scalar case and the case of elasticity: in the first one optimal solutions are related to geodesic transport rays, whereas in the second one we do not even know the connection with any kind of transport problem. The reason is that in general the $\operatorname{class}_{\operatorname{Lip}} \operatorname{Li}_{1, \rho}(\Omega, \Sigma)$ introduced in Sect. 3 cannot be characterized through a two-points condition similar to the one of Proposition 4.2 i). However, the case of Michell energy density (5.7) seems to be an exception: indeed if $\rho(z)=\||| z^{\text {sym }}|| \mid$ we have

$$
u \in \operatorname{Lip}_{1, \rho}\left(\mathbf{R}^{n}\right) \quad \Longleftrightarrow \quad(u(x)-u(y)) \cdot(x-y) \leq|x-y|^{2}
$$

and some of the duality arguments of the scalar case could be applied to this case as well. This matter will be the object of a forthcoming paper.

Acknowledgements. This paper has been written during scientific visits of the two authors to Universities of Pisa and Toulon respectively. The visit of G. Bouchitté to Pisa was financed by italian GNAFA/INDAM, the visit of G. Buttazzo to Toulon was financed by the Université de Toulon et du Var. We wish to thank these institution for the financial support and the warm hospitality. We also acknowledge our colleagues Fréderic Golay and Pierre Seppecher for sharing some numerical computations and mechanical interpretation.

\section{References}

1. Allaire, G., Kohn, R.V.: Optimal design for minimum weight and compliance in plane stress using extremal microstructures. Eur. J. Mech. A/Solids 12(6), 839-878 (1993)

2. Baiocchi, C., Buttazzo, G., Gastaldi, F., Tomarelli, F.: General existence results for unilateral problems in continuum mechanics. Arch. Rational Mech. Anal. 100, 149189 (1988)

3. Bellieud, M., Bouchitté, G.: Homogenization of elliptic problems in a fiber reinforced structure. Nonlocal effects. Ann. Scuola Norm. Sup. Pisa Cl. Sci. 26, 407-436 (1998)

4. Bendsøe, M.: Optimal shape design as a material distribution problem. Struct. Optim. 1, 193-202 (1989)

5. Bouchitté, G., Buttazzo, G., Seppecher, P.: Energies with respect to a measure and applications to low dimensional structures. Calc. Var. 5, 37-54 (1997)

6. Bouchitté, G., Buttazzo, G., Seppecher, P.: Shape optimization solutions via MongeKantorovich equation. C. R. Acad. Sci. Paris 324-I, 1185-1191 (1997)

7. Bouchitté, G., Valadier, M.: Integral representation of convex functionals on a space of measures. J. Funct. Anal. 80, 398-420 (1988)

8. Castaing, C., Valadier, M.: Convex Analysis and Measurable Multifunctions. Lecture Notes Math., Vol. 580. Berlin, Heidelberg, New York: Springer 1977

9. Cea, J., Malanowski, K.: An example of a max-min problem in partial differential equations. SIAM J. Control 8, 305-316 (1970) 
10. Demengel, F.: Déplacements à déformations bornées et champs de contrainte mesures. Ann. Scuola Norm. Sup. Pisa Cl. Sci. 12, 243-318 (1985)

11. Demengel, F., Suquet, P.: On locking materials. Acta Applicandae Math. 6, 185-211 (1986)

12. Ekeland, I., Temam, R.: Convex Analysis and Variational Problems. Studies in Mathematics and its Applications 1. Amsterdam: North-Holland 1976

13. Evans, L.C.: Partial differential equations and Monge-Kantorovich mass transfer. Current Developments in Mathematics, Cambridge MA (1997), 65-126, Boston, MA: Int. Press 1999

14. Evans, L.C., Gangbo, W.: Differential Equations Methods for the Monge-Kantorovich Mass Transfer Problem. Mem. Amer. Math. Soc. 137, Providence 1999

15. Francfort, G.A., Murat, F.: Homogenization and optimal bounds in linear elasticity. Arch. Rational Mech. Anal. 94, 307-334 (1986)

16. Gangbo, W., McCann, R.J.: The geometry of optimal transportation. Acta Math. 177, 113-161 (1996)

17. Goffman, C., Serrin, J.: Sublinear functions of measures and variational integrals. Duke Math. J. 31, 159-178 (1964)

18. Golay, F., Seppecher, P.: Locking materials and topology of optimal shapes. Preprint 2000-11 ANLA. Eur. J. Mech. A/Solids (submitted)

19. Kohn, R.V., Strang, G.: Optimal design and relaxation of variational problems, I, II, III. Comm. Pure Appl. Math. 39, 113-137, 139-182, 353-377 (1986)

20. Mosco, U.: Composite media and asymptotic Dirichlet forms. J. Funct. Anal. 123, 368-421 (1994)

21. Murat, F., Tartar, L.: Calcul des variations et homogénéisation. Proceedings of "Les Méthodes de l'homogénéisation: Théorie et applications en physique", Ecole d'Eté d'Analyse Numérique C.E.A.-E.D.F.-INRIA, Bréau-sans-Nappe 1983, Collection de la direction des études et recherches d'electricité de France 57, Eyrolles, Paris (1985), 319-369

22. Murat, F., Tartar, L.: Optimality conditions and homogenization. Proceedings of "Nonlinear variational problems", Isola d'Elba 1983, Res. Notes Math., Vol. 127, pp. 1-8. London: Pitman 1985

23. Rachev, S.T., Rüschendorf, L.: Mass transportation problems. Vol. I Theory, Vol. II Applications. Probability and its Applications, Berlin, Heidelberg, New York: Springer 1998

24. Tartar, L.: Estimations Fines des Coefficients Homogénéises. Ennio De Giorgi Colloqium, ed. by P. Krée, Res. Notes Math., Vol. 125, pp. 168-187. London: Pitman 1985 